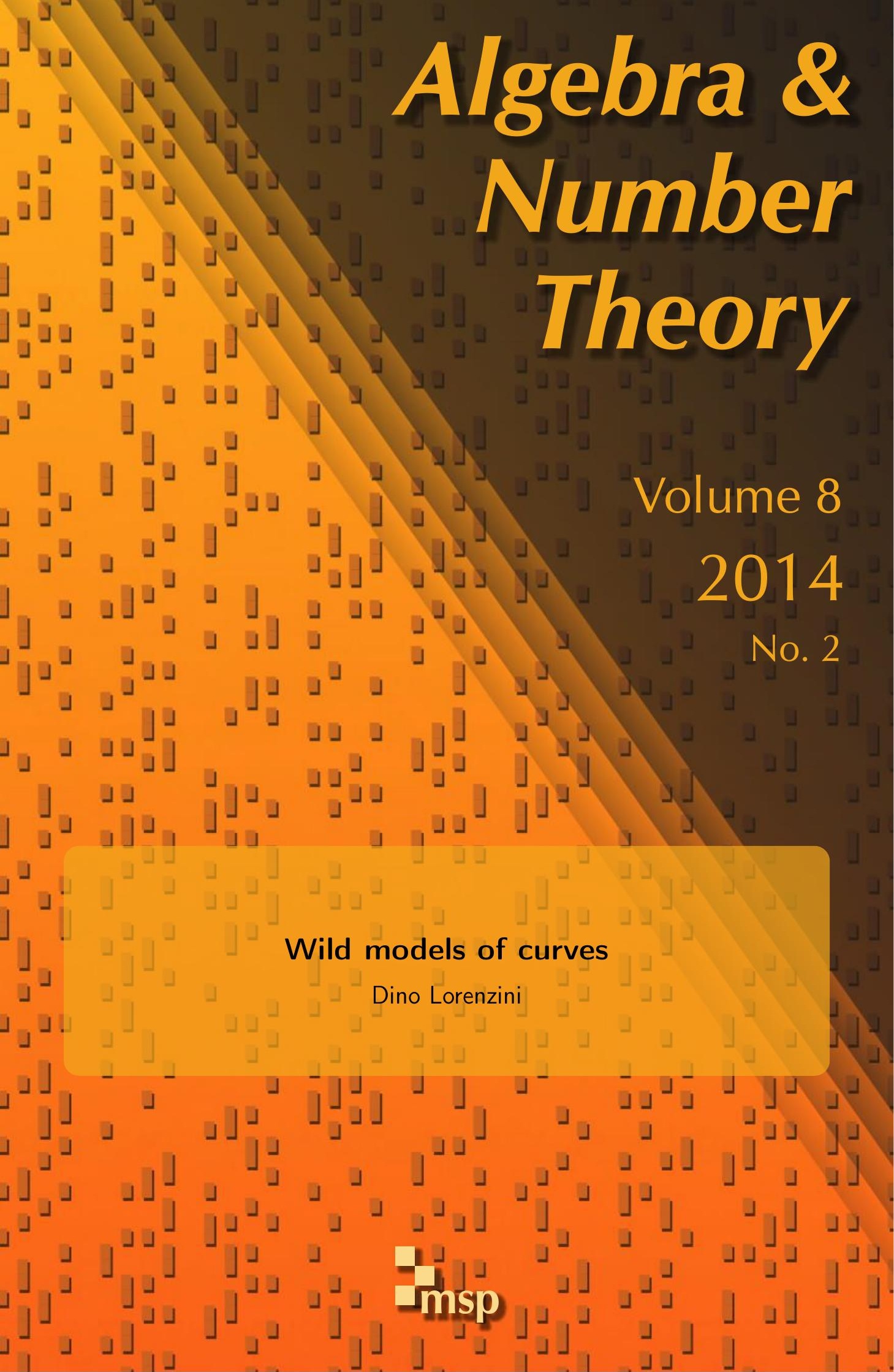




\title{
Wild models of curves
}

\author{
Dino Lorenzini
}

Let $K$ be a complete discrete valuation field with ring of integers $\mathcal{O}_{K}$ and algebraically closed residue field $k$ of characteristic $p>0$. Let $X / K$ be a smooth proper geometrically connected curve of genus $g>0$ with $X(K) \neq \varnothing$ if $g=1$. Assume that $X / K$ does not have good reduction and that it obtains good reduction over a Galois extension $L / K$ of degree $p$. Let $\mathcal{Y} / \mathcal{O}_{L}$ be the smooth model of $X_{L} / L$. Let $H:=\operatorname{Gal}(L / K)$.

In this article, we provide information on the regular model of $X / K$ obtained by desingularizing the wild quotient singularities of the quotient $\mathcal{Y} / \mathrm{H}$. The most precise information on the resolution of these quotient singularities is obtained when the special fiber $\mathcal{Y}_{k} / k$ is ordinary. As a corollary, we are able to produce for each odd prime $p$ an infinite class of wild quotient singularities having pairwise distinct resolution graphs. The information on the regular model of $X / K$ also allows us to gather insight into the $p$-part of the component group of the Néron model of the Jacobian of $X$.

\section{Introduction}

Let $K$ be a complete discrete valuation field with valuation $v$, ring of integers $\mathcal{O}_{K}$ and residue field $k$ of characteristic $p>0$, assumed to be algebraically closed. Let $X / K$ be a smooth proper geometrically connected curve of genus $g>0$ with $X(K) \neq \varnothing$ if $g=1$.

Assume that $X / K$ does not have good reduction and that it obtains good reduction over a Galois extension $L / K$. Let $\mathcal{Y} / \mathcal{O}_{L}$ be the smooth model of $X_{L} / L$. Let $H:=\operatorname{Gal}(L / K)$, and let $\mathcal{Z} / \mathcal{O}_{K}$ denote the quotient $\mathcal{Y} / H$. A regular model for $X / K$ can be obtained by resolving the singularities of the scheme $\mathcal{Z}$. Our goal is to obtain information on this regular model when $p$ divides $[L: K]$. Since the presence of wild ramification renders the subject quite challenging, we will restrict our attention in this article to the case where $[L: K]=p$.

Lorenzini was supported by NSF Grant 0902161.

MSC2010: primary 14G20; secondary 14G17, 14K15, $14 \mathrm{~J} 17$.

Keywords: model of a curve, ordinary curve, cyclic quotient singularity, wild ramification, arithmetical tree, resolution graph, component group, Néron model. 
Beyond our interest in models of curves per se, our motivation for understanding these regular models is twofold. First, since $\mathcal{X}$ is obtained by desingularizing certain quotient singularities, we hope to gain more insight in the general theory of resolutions of wild quotient singularities by producing interesting classes of examples where the singularities can be resolved explicitly. Second, since from a regular model of the curve one can compute much of the Néron model of its Jacobian, we hope to bring new insight into the structure of the rather mysterious $p$-part of the component group of the Néron model of a general abelian variety from an increased understanding of the special case of Jacobians of curves.

Let us introduce some notation needed to state our theorems. Let $\sigma$ denote a generator of $H:=\operatorname{Gal}(L / K)$. Denote also by $\sigma$ the automorphism of $\mathcal{Y}_{k}$ induced by the action of $H$ on $\mathcal{Y}$. The scheme $\mathcal{Z}$ is singular exactly at the images $Q_{1}, \ldots, Q_{d}$ of the ramification points $P_{1}, \ldots, P_{d}$ of the map $\mathcal{Y}_{k} \rightarrow \mathcal{Y}_{k} /\langle\sigma\rangle$ (5.2). Consider the regular model $\mathcal{X} \rightarrow \mathcal{Z}$ obtained from $\mathcal{Z}$ by a minimal desingularization. Let $\mathcal{X}^{\prime} \rightarrow \mathcal{X}$ denote the regular model of $X / K$ minimal with the property that $\mathcal{X}_{k}^{\prime}$ has smooth components and normal crossings. Let $f$ denote the composition $\mathcal{X}^{\prime} \rightarrow \mathcal{Z}$. Let $C_{0} / k$ denote the strict transform in $\mathcal{X}^{\prime}$ of the irreducible closed subscheme $\mathcal{Z}_{k}^{\text {red }}$ of $\mathcal{Z}$. Let $D_{1}, \ldots, D_{d}$ denote the irreducible components of $\mathcal{X}_{k}^{\prime}$ that meet $C_{0}$. Let $r_{i}$ denote the multiplicity of $D_{i}, i=1, \ldots, d$, in $\mathcal{X}_{k}^{\prime}$.

Recall that to any connected curve $\bigcup_{\ell=1}^{n} C_{\ell}$ on a regular model $\mathcal{X}$ we associate a graph $G$ as follows: the vertices are the irreducible components $C_{\ell}$, and in $G$, the vertices $C_{i}$ and $C_{j}(i \neq j)$ are linked by exactly $\left(C_{i} \cdot C_{j}\right) \mathcal{X}$ edges, where $\left(C_{i} \cdot C_{j}\right) \mathcal{X}$ denotes the intersection number of $C_{i}$ and $C_{j}$ on the regular scheme $\mathcal{X}$. Recall that the degree of a vertex $v$ of a graph is the number of edges attached to $v$. A node on a graph is a vertex of degree at least 3 . A vertex of degree 1 is a terminal vertex. A chain is a subgraph of $G$ with vertices $C_{0}, C_{1}, \ldots, C_{n}, n \geq 1$, such that $C_{i}$ is linked to $C_{i+1}$ by exactly one edge in $G$ when $i=0, \ldots, n-1$ and the degree of $C_{i}$ is 2 when $i=1, \ldots, n-1$. If the chain contains a terminal vertex (which can only be $C_{0}$ or $C_{n}$ ), the chain is called a terminal chain.

Let $G$ denote the graph associated with $\mathcal{X}_{k}^{\prime}$. We assume $d \geq 1$. For each $i=1, \ldots, d$, let $G_{Q_{i}}$ denote the graph associated with the curve $f^{-1}\left(Q_{i}\right)$. In particular, $D_{i}$ corresponds to a vertex of $G_{Q_{i}}$. We have the following configuration on the graph $G$ (where a positive integer next to a vertex denotes the multiplicity of the corresponding irreducible component in $\mathcal{X}^{\prime}$ ):

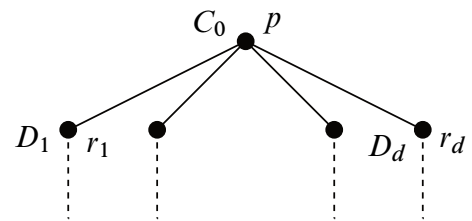


Theorem 5.3. Let $X / K$ be a curve with potentially good reduction after a wildly ramified extension $L / K$ of degree $p$, as above. Keep the above notation. Then, for all $i=1, \ldots, d$, the graph $G_{Q_{i}}$ contains a node of $G$ and $p$ divides $r_{i}$.

In contrast, when $H$ is of prime order $q \neq p$, then it is known that $q>r_{i}$ and that the graph $G_{Q_{i}}$ does not contain a node of $G$. In particular, when $L / K$ is tame and $d \geq 3$, the graph $G$ has only a single node, the component $C_{0}$ (see, e.g., [Lorenzini 1990a, Theorem 2.1]).

We propose in 6.1 a combinatorial measure $\gamma_{Q_{i}} g_{Q_{i}}$ of the complexity of the graph $G_{Q_{i}}$, which we conjecturally relate in 6.2 to the higher ramification data of the morphism $\mathcal{Y}_{k} \rightarrow \mathcal{Y}_{k} /\langle\sigma\rangle$. This conjectural relationship expresses the fact that the graph $G_{Q_{i}}$ is "complicated" only if the higher ramification above $Q_{i}$ is "large". We prove this conjecture in the ordinary case (Theorem 6.4).

Recall that a smooth proper curve $Y / k$ of genus $g$ is called ordinary if its Jacobian $J / k$ is an ordinary abelian variety (that is, $J(k)$ has exactly $p^{g}$ points of order dividing $p$ ). When $\mathcal{Y}_{k}$ is ordinary, the morphism $\mathcal{Y}_{k} \rightarrow \mathcal{Y}_{k} /\langle\sigma\rangle$ has the smallest possible ramification data at each $Q_{i}(2.2)$, and in this case, we can use Theorem 5.3 to describe the graph $G_{Q_{i}}$ explicitly, as in the following theorem, whose statement is slightly strengthened in the version given in Section 6. In the graph below, a bullet $\bullet$ represents an irreducible component of the desingularization of $Q_{i}$. A negative number next to a vertex is the self-intersection of the component. A positive number next to a vertex is the multiplicity of the corresponding component in $\mathcal{X}_{k}^{\prime}$.

Theorem (see Theorem 6.8). Let $X / K$ be a curve with potentially good reduction after a Galois extension $L / K$ of degree $p$, as above. Assume that $\mathcal{Y}_{k}$ ordinary. Then, for all $i=1, \ldots, d$, we have $r_{i}=p$ and $G_{Q_{i}}$ is a graph with a single node $C_{i}$ of degree 3:

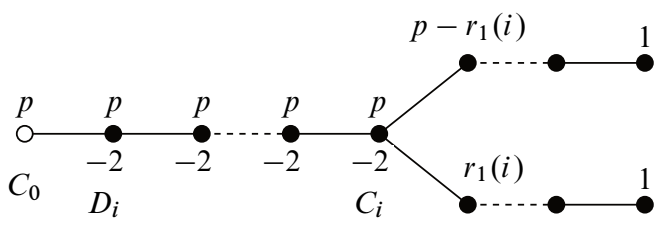

The intersection matrix $N\left(p, \alpha_{i}, r_{1}(i)\right)$ of the resolution of $Q_{i}$ is uniquely determined as in 4.7 by the two integers $\alpha_{i}$ and $r_{1}(i)$ with $1 \leq r_{1}(i)<p$. The integer $\alpha_{i}$ denotes the number of vertices of self-intersection -2 (including the node $C_{i}$ ) on the chain in $G_{Q_{i}}$ connecting the node $C_{0}$ to the single node $C_{i}$ of $G_{Q_{i}}$, and the integer $\alpha_{i}$ is divisible by $p$.

To further determine the regular model, one would need to determine explicitly the integers $\alpha_{i}$ and $r_{1}(i)$. We address this issue in [Lorenzini 2013b]. In all cases where we have been able to compute $\alpha_{i}$ and $r_{1}(i)$, we found them to be related to the valuation of the different of $L / K$. More precisely, let $\left(s_{L / K}+1\right)(p-1)$ 
denote the valuation of the different of $L / K$. In [Lorenzini 2013b, Theorem 1.1], we present some instances where $\alpha_{i}=p s_{L / K}$ and $r_{1}(i) \equiv-s_{L / K}^{-1}$ modulo $p$. We also show in [Lorenzini 2013b, Theorem 4.1] that the singularities $Q_{i}$ are rational.

Remark 1.1. The same type of intersection matrix, $N\left(p, \alpha_{i}, r_{1}(i)\right)$, also occurs in the resolution of the singularities of the model $\mathcal{Z}$ when $X / K$ has genus $p-1$ and $\operatorname{Jac}(X) / K$ has purely toric reduction after an extension of degree $p$ [Lorenzini 2010, Theorem 2.2].

Remark 1.2. The special fiber of the model $\mathcal{X} / \mathcal{O}_{K}$ of $X / K$ in Theorem 6.8 has thus a graph with a central vertex to which $d$ branches are attached, of the form described below, where we picture the case $d=4$.

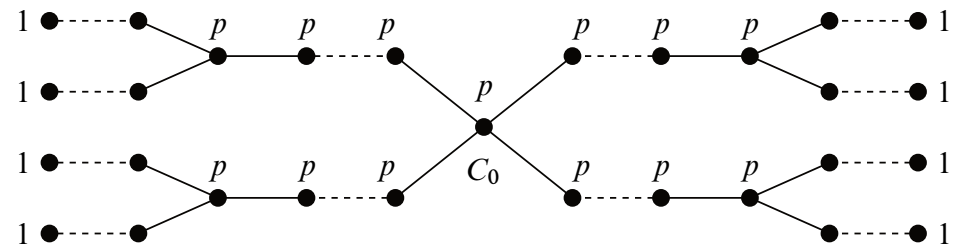

Fix any $d>1$. We establish in Theorem 6.8 and Example 6.13 the existence of some field $K$ of residue characteristic $p>0$ and of some smooth proper curve $X / K$ with a regular model whose special fiber has a graph of the above type. This is clearly a weak existence result, but our understanding of models in the presence of wild ramification is so limited that even this weak existence result does not follow from the general existence results of Viehweg [1977] and Winters [1974].

An immediate but surprising corollary to Theorem 6.8 is as follows.

Corollary (see Corollary 6.10). Let $X / K$ be a curve of genus $g>1$ with potentially good reduction after a Galois extension $L / K$ of degree $p$, as above. Assume that $\mathcal{Y}_{k}$ is ordinary. Then $X(K) \neq \varnothing$.

The information on the regular model of $X / K$ obtained in Theorem 6.8, while incomplete to fully describe the special fiber of the model, suffices to compute several invariants of arithmetical interest. For instance, the set of components of multiplicity 1 on the special fiber of the model is determined, and this information is one of the ingredients needed to apply the Chabauty-Coleman method to bound the number of $\mathbb{Q}$-rational points on a curve $X / \mathbb{Q}$ using the reduction at a small prime $p$, as in [Lorenzini and Tucker 2002, Theorem 1.1]. Let $A / K$ denote the Jacobian of $A / K$ with Néron model $\mathcal{A} / \mathcal{O}_{K}$ and component group $\Phi_{A / K}$. The information obtained in Theorem 6.8 suffices to compute $\Phi_{A / K}$ and a new canonical subgroup $\Phi_{A / K}^{0}$ of $\Phi_{A / K}$ that we now define. 
1.3. Let $A / K$ be an abelian variety with Néron model $\mathcal{A} / \mathcal{O}_{K}$. Let $L / K$ be any finite extension, and let $\mathcal{A}^{\prime} / \mathcal{O}_{L}$ denote the Néron model of $A_{L} / L$. Denote by

$$
\eta: \mathcal{A} \times \mathcal{O}_{K} \mathcal{O}_{L} \rightarrow \mathcal{A}^{\prime}
$$

the canonical map induced by the functoriality property of Néron models. The special fiber $\mathcal{A}_{k}$ is an extension of a finite group $\Phi_{A / K}$, called the group of components, by the connected component of zero $\mathcal{A}_{k}^{0}$ of $\mathcal{A}_{k}$ :

$$
0 \rightarrow \mathcal{A}_{k}^{0} \rightarrow \mathcal{A}_{k} \rightarrow \Phi_{A / K} \rightarrow 0 .
$$

Assume that $A_{L} / L$ has semistable reduction, and consider the natural map $\Phi_{A / K} \rightarrow \mathcal{A}_{k}^{\prime} / \eta\left(\mathcal{A}_{k}^{0}\right)$. We let

$$
\Phi_{A / K}^{0}:=\operatorname{Ker}\left(\Phi_{A / K} \rightarrow \mathcal{A}_{k}^{\prime} / \eta\left(\mathcal{A}_{k}^{0}\right)\right) \text {. }
$$

The subgroup $\Phi_{A / K}^{0}$ does not depend on the choice of such an extension $L / K$ and is functorial in $A$. Our interest in this subgroup stems from the following conjectures.

When $A / K$ has potentially good reduction and, more generally, when the toric rank of $\mathcal{A}_{k}^{0}$ is trivial, we conjecture that the order of the group $\Phi_{A / K}$ is bounded by a constant depending only on the dimension $g$ of $A / K$ [Lorenzini 1990b, p. 146]. This statement is true when $A / K$ is a Jacobian [Lorenzini 1990b, Theorem 2.4] and for the prime-to- $p$ part of $\Phi_{A / K}$ [Lorenzini 1993, Theorem 2.15]. Since $[L: K]^{2}$ kills the group $\Phi_{A / K}$ when the toric rank of $\mathcal{A}$ is trivial [Liu and Lorenzini 2001, Proposition 1.8], we find that, to prove the conjecture that $\Phi_{A / K}$ is bounded by a constant depending only on $g$, it suffices to prove that the minimal number of generators of $\Phi_{A / K}$ can be bounded by a constant depending on $g$ only. We guess, under the above hypotheses, that $\Phi_{A / K}$ can be generated by $2 g$ elements.

Assume now that $A / K$ has potentially good reduction. The $p$-torsion in $\mathcal{A}_{k}^{\prime}$ can always be generated by at most $g$ elements. Thus, the above conjecture is proved if the $p$-part of the kernel $\Phi_{A / K}^{0}$ can be generated by a number of elements bounded by a constant depending on $g$ only (possibly $2 g$ ). In the ordinary case, where the $p$-torsion in $\mathcal{A}_{k}^{\prime}$ is minimally generated by $g$ elements, one may wonder if $\Phi_{A / K}^{0}$ can also be generated by $g$ elements. Our next corollary gives some evidence that this latter question may have a positive answer for all abelian varieties with potentially good ordinary reduction.

Let $A / K$ be the Jacobian of a curve $X / K$ with $X(K) \neq \varnothing$. We denote by $\langle\cdot, \cdot\rangle: \Phi_{A / K} \times \Phi_{A / K} \rightarrow \mathbb{Q} / \mathbb{Z}$ Grothendieck's pairing, which is nondegenerate [Bosch and Lorenzini 2002, Theorem 4.6]. We denote by $\left(\Phi_{A / K}^{0}\right)^{\perp}$ the orthogonal of $\Phi_{A / K}^{0}$ under Grothendieck's pairing.

Corollary (see Corollary 6.12). Let $A / K$ be the Jacobian of a curve $X / K$ of genus $g>1$ having potentially good ordinary reduction after a Galois extension $L / K$ of degree $p$, as above. Then $\Phi_{A / K}$ is a $\mathbb{Z} / p \mathbb{Z}$-vector space of dimension $2 d-2$, and $\Phi_{A / K}^{0}$ is a subspace of dimension $d-1$. Moreover, $\Phi_{A / K}^{0}=\left(\Phi_{A / K}^{0}\right)^{\perp}$. 
It is natural in view of Corollary 6.12 to wonder whether the same result holds for all principally polarized abelian varieties $A / K$ having potentially good ordinary reduction after a Galois extension $L / K$ of degree $p$. We may also wonder, for any principally polarized abelian variety $A / K$ with potentially good reduction, whether the order of $\Phi_{A / K}^{0} \cap\left(\Phi_{A / K}^{0}\right)^{\perp}$ can be bounded by a constant depending only on the $p$-rank of $\mathcal{A}_{k}^{\prime}$. We hope to return to these questions in the future.

1.4. Our explicit computation of a regular model of a curve having potentially good ordinary reduction also has an application to quotient singularities. Our current understanding of wild $\mathbb{Z} / p \mathbb{Z}$-quotient singularities of surfaces is quite limited, and few explicit examples are known (see, e.g., [Artin 1975], [Katsura 1978] for $p=2$ and [Peskin 1983] for $p=3$ ). In contrast to the case of a tame cyclic quotient singularity, where the number of possible resolution graphs is finite once the order of the group is fixed, we show below that, for any fixed odd prime $p$, there are infinitely many graphs that can occur as the resolution graphs of a wild $\mathbb{Z} / p \mathbb{Z}$ quotient singularity in both mixed characteristic and in the equicharacteristic case. The analogous result when $p=2$ is discussed in [Lorenzini 2013a, Theorem 4.1].

Corollary 6.14. Fix any odd prime $p$. For each integer $m>0$, there exist a 2dimensional regular local ring $B$ of equicharacteristic $p$ endowed with an action of $H:=\mathbb{Z} / p \mathbb{Z}$ and a 2-dimensional regular local ring $B^{\prime}$ of mixed characteristic $(0, p)$ endowed with an action of $\mathbb{Z} / p \mathbb{Z}$ such that $\operatorname{Spec} B^{H}$ and $\operatorname{Spec}\left(B^{\prime}\right)^{H}$ are singular exactly at their closed point and the graphs associated with a minimal resolution of Spec $B^{H}$ and $\operatorname{Spec}\left(B^{\prime}\right)^{H}$ have one node and more than $m$ vertices.

This article is organized as follows. The proof of Theorem 5.3, in Section 5, is of a global nature and includes in particular a study of the natural map $\Phi_{A / K} \rightarrow$ $\mathcal{A}_{k}^{\prime} / \eta\left(\mathcal{A}_{k}^{0}\right)$. The proof uses two auxiliary results of independent interest. The first result, Proposition 2.5, is discussed in Section 2 and is a relation between torsion points in a quotient of two Jacobians. This proposition is one place in our arguments where the tame and wild cases can be seen to differ in an explicit way. The second result, Proposition 3.5, is the main result of Section 3 and is a general relation between elements in the component group $\Phi_{M}$ of an arithmetical tree.

Section 4 presents further results of a combinatorial nature on arithmetical trees that are needed in the proof of Theorem 6.8. Section 6 contains the proof of Theorem 6.8 and of its applications.

\section{Cyclic morphisms and torsion}

Let $k$ be an algebraically closed field of characteristic $p$. Let $f: D \rightarrow C$ be a ramified Galois morphism of smooth connected projective curves over $k$. Our main result in this section is Proposition 2.5, which will be applied to the case of the quotient morphism $\mathcal{Y}_{k} \rightarrow \mathcal{Y}_{k} /\langle\sigma\rangle$ in the course of the proof of Theorem 5.3. 
2.1. Assume that the Galois group $H$ of $f$ is cyclic of degree $q^{s}$ with $q$ prime. Let $P_{1}, \ldots, P_{d}$ in $D(k)$ be the ramification points. Assume that, at each $P_{i}$, the morphism is totally ramified, and let $Q_{i}:=f\left(P_{i}\right), i=1, \ldots, d$, be the branch points.

When $q \neq p$, the Riemann-Hurwitz formula is

$$
2 g(D)-2=q^{s}(2 g(C)-2)+d\left(q^{s}-1\right) .
$$

Moreover, $d \geq 2$. When $g(C)=0$, this follows immediately from the formula; the general case requires a separate proof.

Assume now that $q=p$. For $P \in D(k)$, let $H_{0}(P) \supseteq H_{1}(P) \supseteq \cdots$ denote the sequence of higher ramification groups. If $P$ is a ramification point, then $\left|H_{0}(P)\right|=\left|H_{1}(P)\right|=p^{s}$. Set

$$
\delta(P):=\sum_{i}\left(\left|H_{i}(P)\right|-1\right) .
$$

Then the Riemann-Hurwitz formula is

$$
2 g(D)-2=p^{s}(2 g(C)-2)+\sum_{P \in D(k)} \delta(P)
$$

and it may happen that $d=1$.

2.2. Let $\gamma(D)$ denote the $p$-rank of $D$ (i.e., the $p$-rank of $\operatorname{Jac}(D))$. The DeuringShafarevich formula relates the $p$-ranks of $C$ and $D$ :

$$
\gamma(D)-1=p^{s}(\gamma(C)-1)+d\left(p^{s}-1\right) .
$$

The curve $D$ is ordinary when $\gamma(D)=g(D)$. When $D$ is ordinary, we find, comparing the formulas (2.1.2) and (2.2.1), that $\left|H_{2}(P)\right|=1$ for all $P$ and that $C$ is also ordinary. Moreover, when $g(D)>0$, Equation (2.2.1) shows that $p \leq g(D)+1$.

When a ramification point $P$ of a Galois morphism $f: D \rightarrow C$ is such that $H_{2}(P)=(0)$, we will say that the morphism is weakly ramified at $P$.

2.3. We record here the following well-known fact (see [Hasse 1934, p. 42], or [Singh 1974, Lemma 1.3], when $K=k(x))$. Let $K$ be a field with $\operatorname{char}(K)=p$. Let $(A, \mathcal{M})$ be a discrete valuation ring with field of fractions $K$, valuation $v_{K}$ and uniformizer $\pi_{K}$. Assume that the residue field $k$ of $A$ is algebraically closed. Let $L / K$ be a cyclic ramified Galois extension of degree $p$ with Galois group $H$. Let $(B, \mathcal{N})$ denote the integral closure of $A$ in $L$. Let $H=H_{0} \supseteq H_{1} \supseteq \cdots$ denote the sequence of ramification groups. Then $\sum_{i=0}^{\infty}\left(\left|H_{i}\right|-1\right)=(m+1)(p-1)$ for some integer $m$ prime to $p$. 
2.4. Examples of curves with an automorphism of degree $p$ in characteristic $p$ can be given in Artin-Schreier form. Consider the curve $y^{p}-y=\prod_{i=1}^{d}\left(x-a_{i}\right)^{-n_{i}}$, where $a_{1}, \ldots, a_{s} \in k$ are distinct and the $n_{i}$ are positive integers coprime to $p$. The automorphism $y \mapsto y+1$ has order $p$. The genus $g$ of the smooth complete curve defined by the above equation is given by the Riemann-Hurwitz formula $2 g-2=-2 p+(p-1)\left(\sum_{i=1}^{d}\left(n_{i}+1\right)\right)$ (see [Subrao 1975, p. 8]).

The following simple proposition exhibits a key difference between the tame and wild cases:

Proposition 2.5. Let $q$ be a prime. Let $f: D \rightarrow C$ be a ramified cyclic morphism of degree $q^{s}$ between smooth connected projective curves over $k$. Let $P_{1}, \ldots, P_{d}$, $d \geq 2$, denote the ramification points, assumed to be totally ramified. For $i \neq j$, the image $\omega_{i j}$ of $P_{i}-P_{j}$ in $\operatorname{Jac}(D) / f^{*}(\operatorname{Jac}(C))$ is of finite order $q^{s}$. Let $T$ denote the finite subgroup $\operatorname{Jac}(D) / f^{*}(\operatorname{Jac}(C))$ generated by $\left\{\omega_{i d} \mid i=1, \ldots, d-1\right\}$.

(a) If $q=p$, then $T$ is isomorphic to $\left(\mathbb{Z} / p^{s} \mathbb{Z}\right)^{d-1}$ and is generated by the set $\left\{\omega_{i d} \mid i=1, \ldots, d-1\right\}$.

(b) If $q \neq p$, then $T$ is isomorphic to $\left(\mathbb{Z} / q^{s} \mathbb{Z}\right)^{d-2}$ and is generated by the set $\left\{\omega_{i d} \mid i=1, \ldots, d-2\right\}$.

Proof. Let $S$ denote the subgroup of $\operatorname{Div}^{0}(D)$ with support on the set $\left\{P_{1}, \ldots, P_{d}\right\}$. It is clear that $\left\{P_{i}-P_{d} \mid i=1, \ldots, d-1\right\}$ is a $\mathbb{Z}$-basis for $S$. Let $S \rightarrow T$ denote the natural surjective map. This map factors through $S / q^{s} S$ since $q^{s}\left(\sum_{i} b_{i} P_{i}\right)=$ $f^{*}\left(\sum_{i} b_{i} Q_{i}\right)$ with $\sum_{i} b_{i} Q_{i} \in \operatorname{Div}^{0}(C)$.

Let $\sigma$ be a generator of $\operatorname{Aut}(D / C)$. Suppose that $\sigma\left(\operatorname{div}_{D}(g)\right)=\operatorname{div}_{D}(g)$ for some $g \in k(D)^{*}$. Then $g^{\sigma}=c g$ for some $c \in k^{*}$. Since $\sigma$ has finite order $q^{s}$, we find that $c^{q^{s}}=1$.

Consider first the case where $q=p$. Then $c=1$. Thus, $g^{\sigma}=g$ and $g \in k(C)^{*}$. Suppose that the divisor $\left(\sum_{i} b_{i} P_{i}\right)$ has trivial image in $T$. Then it is possible to write $\left(\sum_{i} b_{i} P_{i}\right)=f^{*}\left(\sum_{j} R_{j}\right)+\operatorname{div}_{D}(h)$ for some $R_{j} \in C(k)$ and $h \in k(D)^{*}$. Then we have $\sigma\left(\operatorname{div}_{D}(h)\right)=\operatorname{div}_{D}(h)$, and we conclude that $h \in k(C)^{*}$. Therefore, we have an equality of divisors of the form $\left(\sum_{i} b_{i} P_{i}\right)=f^{*}(E)$ for some $E \in \operatorname{Div}^{0}(C)$. It follows that $E=\sum_{i} c_{i} Q_{i}$ for some $c_{i}$. Hence, the map $S / p^{s} S \rightarrow T$ is an isomorphism, proving Part (a).

Suppose now that $q \neq p$. Fix a primitive $q^{s}$-th root $\xi$ of 1 . Then $k(D) / k(C)$ is a Kummer extension, generated by the root $\alpha$ of $y^{q^{s}}-a \in k(C)[y]$ such that $\alpha^{\sigma}=\xi \alpha$. It is easy to check that, for each $i=0, \ldots, q^{s}-1$,

$$
\left\{\beta \in k(D) \mid \beta^{\sigma}=\xi^{i} \beta\right\}=k(C) \alpha^{i} .
$$

The equality $\alpha^{\sigma}=\xi \alpha$ implies that $\operatorname{div}_{D}(\alpha)$ can be written as

$$
\left(\sum_{i=1}^{d} a_{i} P_{i}\right)+\sum_{j} c_{j}\left(\sum_{i=0}^{q^{s}-1} \sigma^{i}\left(S_{j}\right)\right)
$$


for some integers $a_{i}$ and $c_{j}$, and some $S_{j} \in D(k) \backslash\left\{P_{1}, \ldots, P_{d}\right\}$. It follows that $q^{s}$ divides $\sum_{i=1}^{d} a_{i}$ since $\operatorname{deg}\left(\operatorname{div}_{D}(\alpha)\right)=0$. This means that the divisor

$$
\sum_{j} c_{j}\left(\sum_{i=0}^{q^{s}-1} \sigma^{i}\left(S_{j}\right)\right)+\left(\sum_{i=1}^{d} a_{i}\right) P_{d}
$$

defines an element in $f^{*}(\operatorname{Jac}(C))$. Hence, the image $v$ of $\left(\sum_{i} a_{i} P_{i}\right)-\left(\sum_{i} a_{i}\right) P_{d}$ in $T$ is trivial. We thus have a map

$$
\varphi: S /\left\langle q^{s} S,\left(\sum_{i} a_{i} P_{i}\right)-\left(\sum_{i} a_{i}\right) P_{d}\right\rangle \rightarrow T .
$$

Let us note that $\left(\sum_{i} a_{i} P_{i}\right)-\left(\sum_{i} a_{i}\right) P_{d} \notin q^{s} S$ because, otherwise, the morphism $f$ given by the Kummer equation $y^{q^{s}}-a$ would not be totally ramified at $P_{1}, \ldots, P_{d}$.

Suppose that the divisor $\left(\sum_{i} b_{i} P_{i}\right)$ has trivial image in $T$. Then it is possible to write $\left(\sum_{i} b_{i} P_{i}\right)=f^{*}\left(\sum_{j} R_{j}\right)+\operatorname{div}_{D}(h)$ for some $R_{j} \in C(k)$ and $h \in k(D)^{*}$. Thus, we have $\sigma\left(\operatorname{div}_{D}(h)\right)=\operatorname{div}_{D}(h)$, and we conclude that $h^{\sigma}=\xi^{j} h$ for some $j \in$ $\left\{0, \ldots, q^{s}-1\right\}$. Therefore, there exists $b \in k(C)^{*}$ such that $h=b \alpha^{j}$. Hence, we have an equality of divisors of the form $\left(\sum_{i} b_{i} P_{i}\right)=f^{*}(E)+j\left[\left(\sum_{i} a_{i} P_{i}\right)-\left(\sum_{i} a_{i}\right) P_{d}\right]$ for some $E \in \operatorname{Div}^{0}(C)$. It follows that $E=\sum_{i} c_{i} Q_{i}$ for some $c_{i}$. Hence, the map $\varphi$ is an isomorphism, proving Part (b).

Corollary 2.6. Assume that $p \neq 2$. Let $D / k$ be a smooth projective connected hyperelliptic curve of genus $g$. Denote by $\tau$ the hyperelliptic involution. Let $\sigma$ be an automorphism of order $p$. Then either $\sigma$ has a single fixed point, fixed by $\tau$, or it has exactly two fixed points, permuted by $\tau$.

Proof. The hyperelliptic involution commutes with $\sigma$, and hence, it permutes the fixed points $\left\{P_{1}, \ldots, P_{d}\right\}$. If $d \geq 2$ and two fixed points $P_{1}$ and $P_{2}$ of $\sigma$ are fixed by $\tau$, then the divisor class $P_{1}-P_{2}$ is fixed by $\tau$. Proposition 2.5 shows that the class of $P_{1}-P_{2}$ is not trivial and, since $p>2$, this divisor class is not equal to the class of $-\left(P_{1}-P_{2}\right)$. This is a contradiction since $\tau$ acts as the [-1]-map on $\operatorname{Jac}(D)$. Thus, $\tau$ fixes at most one point $P_{i}$.

If $d \geq 3$, then we may assume that either $\tau\left(P_{1}\right)=P_{2}$ and $P_{3}$ is fixed or that $\tau\left(P_{1}\right)=P_{2}$ and $\tau\left(P_{3}\right)=P_{4}$. In the first case, we find that $\tau\left(P_{1}-P_{3}\right)=\left(P_{2}-P_{3}\right)=$ $-\left(P_{1}-P_{2}\right)+\left(P_{1}-P_{3}\right)$. Using the fact that $\tau$ acts as the [-1]-map on $\operatorname{Jac}(D)$, we find the relation $-\left(P_{1}-P_{3}\right)=-\left(P_{1}-P_{2}\right)+\left(P_{1}-P_{3}\right)$ in $\operatorname{Jac}(D)$. Looking at this relation in $T$ contradicts Proposition 2.5. The other case is similar and is left to the reader.

Example 2.7. Assume that $p \neq 2$. Consider a smooth hyperelliptic curve $C / k$ given by an affine equation $y^{2}=f(x)$, and let $D$ be its Galois cover given by the equation $z^{p}-z=x$. The automorphism $\sigma: D \rightarrow D$ with $\sigma(z)=z+1$ has one fixed point $P$ with $\delta(P)=3(p-1)$ when $\operatorname{deg}(f)$ is odd, and it has two fixed points $P_{1}$ and $P_{2}$ with $\delta\left(P_{1}\right)=\delta\left(P_{2}\right)=2(p-1)$ when $\operatorname{deg}(f)$ is even. 


\section{Arithmetical trees}

Our main result in this section is Proposition 3.5, which will be needed in the proof of Theorem 5.3. This proposition pertains to arithmetical graphs, and we now recall how one associates such an object to any regular model of a curve.

Let $X / K$ be any smooth, proper, geometrically connected curve of genus $g$. Let $\mathcal{X} / \mathcal{O}_{K}$ be a regular model of $X / K$. Let $\mathcal{X}_{k}:=\sum_{i=1}^{v} r_{i} C_{i}$ denote the special fiber of $\mathcal{X}$, where $C_{i}$ is an irreducible component and $r_{i}$ is its multiplicity. Let $M:=\left(\left(C_{i} \cdot C_{j}\right)\right)_{1 \leq i, j \leq v}$ be the associated intersection matrix. Denote by $G$ the associated graph. Let ${ }^{t} R:=\left(r_{1}, \ldots, r_{v}\right)$ so that $M R=0$. We call the triple $(G, M, R)$ an arithmetical graph (in [Lorenzini 1989], the additional condition that $\operatorname{gcd}\left(r_{1}, \ldots, r_{v}\right)=1$ is assumed, and it is $(G,-M, R)$ that is called an arithmetical graph). For the purpose of simplifying the statements of some definitions, we sometimes think of $G$ as a metric space with the natural topology where each edge of $G$ with its two endpoints is homeomorphic to the closed unit interval $[0,1]$.

Let $(G, M, R)$ be any arithmetical graph on $v$ vertices. Let $M: \mathbb{Z}^{v} \rightarrow \mathbb{Z}^{v}$ and ${ }^{t} R: \mathbb{Z}^{v} \rightarrow \mathbb{Z}$ be the linear maps associated to the matrices $M$ and $R$. The group of components of $(G, M, R)$ is defined as

$$
\Phi_{M}:=\operatorname{Ker}\left({ }^{t} R\right) / \operatorname{Im}(M)=\left(\mathbb{Z}^{v} / \operatorname{Im}(M)\right)_{\text {tors }} .
$$

Motivated by the case of degenerations of curves, we shall denote by $(C, r(C))$ a vertex of $G$, where $r(C)$ is the coefficient of $R$ corresponding to $C$. The integer $r(C)$, also denoted simply by $r$, is called the multiplicity of $C$. The matrix $M$ is written as $M:=\left(\left(C_{i} \cdot C_{j}\right)\right)_{1 \leq i, j \leq v}$, and we write $\left|C_{i} \cdot C_{i}\right|:=\left|\left(C_{i} \cdot C_{i}\right)\right|$.

3.1. Denote by $\langle\cdot, \cdot\rangle: \Phi_{M} \times \Phi_{M} \rightarrow \mathbb{Q} / \mathbb{Z}$ the perfect pairing $\langle\cdot, \cdot\rangle_{M}$ attached in [Bosch and Lorenzini 2002, Lemma 1.1] to the symmetric matrix $M$. Explicit values of this pairing are computed as follows. Let $(C, r)$ and $\left(C^{\prime}, r^{\prime}\right)$ be two distinct vertices of $G$. Define

$$
E\left(C, C^{\prime}\right):={ }^{t}\left(0, \ldots, 0, \frac{r^{\prime}}{\operatorname{gcd}\left(r, r^{\prime}\right)}, 0, \ldots, 0, \frac{-r}{\operatorname{gcd}\left(r, r^{\prime}\right)}, 0, \ldots, 0\right) \in \mathbb{Z}^{v},
$$

where the first nonzero coefficient of $E\left(C, C^{\prime}\right)$ is in the column corresponding to the vertex $C$ and, similarly, the second nonzero coefficient is in the column corresponding to the vertex $C^{\prime}$. We say that the pair $\left(C, C^{\prime}\right)$ is uniquely connected if there exists a path $\mathcal{P}$ in $G$ between $C$ and $C^{\prime}$ such that, for each edge $e$ on $\mathcal{P}$, the graph $G \backslash\{e\}$ is disconnected. Note that, when a pair $\left(C, C^{\prime}\right)$ is uniquely connected, then the path $\mathcal{P}$ is the unique shortest path between $C$ and $C^{\prime}$. A graph is a tree if and only if every pair of vertices of $G$ is uniquely connected.

Let $(C, r)$ and $\left(C^{\prime}, r^{\prime}\right)$ be a uniquely connected pair with associated path $\mathcal{P}$. While walking on $\mathcal{P} \backslash\left\{C, C^{\prime}\right\}$ from $C$ to $C^{\prime}$, label each encountered vertex consecutively 
by $\left(C_{1}, r_{1}\right),\left(C_{2}, r_{2}\right), \ldots,\left(C_{n}, r_{n}\right)$. Let $G_{i}$ denote the connected component of $C_{i}$ in $G \backslash$ edges of $\mathcal{P}\}$. The graph $G_{i}$ is reduced to a single vertex if and only if $C_{i}$ is not a node of $G$. For convenience, we write $(C, r)=\left(C_{0}, r_{0}\right)$ and $\left(C^{\prime}, r^{\prime}\right)=\left(C_{n+1}, r_{n+1}\right)$ and define $G_{0}$ and $G_{n+1}$ accordingly.

3.2. The following facts are proved in [Bosch and Lorenzini 2002, Proposition 5.1]. Let $(G, M, R)$ be any arithmetical graph. Let $C$ and $C^{\prime}$ be two vertices such that $\left(C, C^{\prime}\right)$ is a uniquely connected pair of $G$. Let $\gamma$ denote the image of $E\left(C, C^{\prime}\right)$ in $\Phi_{M}$. For $(D, s)$ and $\left(D^{\prime}, s^{\prime}\right)$ any two distinct vertices on $G$, let $\delta$ denote the image of $E\left(D, D^{\prime}\right)$ in $\Phi_{M}$. Writing $\mathcal{P}$ for the oriented shortest path from $C$ to $C^{\prime}$ as above, let $C_{\alpha}$ denote the vertex of $\mathcal{P}$ closest to $D$ in $G$, and let $C_{\beta}$ denote the vertex of $\mathcal{P}$ closest to $D^{\prime}$. In other words, $D \in G_{\alpha}$ and $D^{\prime} \in G_{\beta}$. Assume that $\alpha \leq \beta$. (Note that we may have $\alpha=\beta$, and we may have $D=C_{\alpha}$ or $D^{\prime}=C_{\beta}$.) Then if $\alpha<\beta$,

$$
\langle\gamma, \delta\rangle=-\operatorname{lcm}\left(r, r^{\prime}\right) \operatorname{lcm}\left(s, s^{\prime}\right)\left(\frac{1}{r_{\alpha} r_{\alpha+1}}+\frac{1}{r_{\alpha+1} r_{\alpha+2}}+\cdots+\frac{1}{r_{\beta-1} r_{\beta}}\right)
$$

$\bmod \mathbb{Z}$,

and if $C_{\alpha}=C_{\beta}$, then $\langle\gamma, \delta\rangle=0$. Moreover,

$$
\langle\gamma, \gamma\rangle=-\operatorname{lcm}\left(r, r^{\prime}\right)^{2}\left(\frac{1}{r r_{1}}+\frac{1}{r_{1} r_{2}}+\cdots+\frac{1}{r_{n} r^{\prime}}\right) \quad \bmod \mathbb{Z} .
$$

Note that the negative signs in the expressions (3.2.1) and (3.2.2) are missing in [Bosch and Lorenzini 2002, Proposition 5.1]. Thus, all expressions for $\langle\gamma, \delta\rangle$ computed in Section 5 of [Bosch and Lorenzini 2002] using Proposition 5.1 are correct only after having been multiplied by -1 . Similar sign mistakes occurred in [Lorenzini 2000]. The proof of [Bosch and Lorenzini 2002, Proposition 5.1] is correct except that its last line produces the opposite of the stated values for $\langle\gamma, \delta\rangle$ since we assume $\alpha \leq \beta$.

3.3. Let $(C, r)$ be a vertex of $G$ of degree $d \geq 2$. Let $\left(D_{i}, r_{i}\right), i=1, \ldots, d$, denote the neighbors of $C$, that is, the vertices of $G$ linked to $C$. Let $\tau_{i}$ denote the image of $E\left(D_{i}, D_{d}\right)$ in $\Phi_{M}$ for $i \in\{1, \ldots, d-1\}$. We will use repeatedly the following expressions computed using (3.2.1) and (3.2.2):

$$
\left\langle\tau_{i}, \tau_{i}\right\rangle=-\operatorname{lcm}\left(r_{i}, r_{d}\right)^{2} \frac{r_{i}+r_{d}}{r_{i} r_{d} r} \bmod \mathbb{Z}
$$

and, when $i \neq j$,

$$
\left\langle\tau_{i}, \tau_{j}\right\rangle=-\operatorname{lcm}\left(r_{i}, r_{d}\right) \operatorname{lcm}\left(r_{j}, r_{d}\right) \frac{1}{r_{d} r} \bmod \mathbb{Z} .
$$

These formulas allow us to easily show that $\tau_{i}$ may not always be trivial. For example, let $p$ be a prime dividing $r$. When $p \nmid r_{i} r_{d}\left(r_{i}+r_{d}\right)$, we find that $\left\langle\tau_{i}, \tau_{i}\right\rangle \neq 0$ 
and, thus, $\tau_{i} \neq 0$. Similarly, when for three distinct indices $i, j$ and $d$ we have $p \nmid r_{i} r_{j} r_{d}$, we find that $\left\langle\tau_{i}, \tau_{j}\right\rangle \neq 0$, showing that both $\tau_{i}$ and $\tau_{j}$ are not trivial.

We claim that $r$ kills $\tau_{i}$. Indeed, we find, using [Lorenzini 2000, Lemma 2.2], that the images in $\Phi_{M}$ of $E\left(D_{i}, C\right)$ and $E\left(C, D_{d}\right)$ have order dividing $\operatorname{gcd}\left(r_{i}, r\right)$ and $\operatorname{gcd}\left(r, r_{d}\right)$, respectively. Consider the following easy relation between vectors in $\mathbb{Z}^{v}$ [Lorenzini 2000, Remark 3.5]: given any three vertices $(A, a),(B, b)$ and $(C, c)$,

$$
b E(A, C)=\frac{c}{\operatorname{gcd}(a, c)} \operatorname{gcd}(a, b) E(A, B)+\frac{a}{\operatorname{gcd}(a, c)} \operatorname{gcd}(b, c) E(B, C) .
$$

Using this relation, we find that $r \tau_{i}=0$.

Lemma 3.4. Let $(G, M, R)$ be an arithmetical graph. Consider any two distinct vertices $(A, a)$ and $\left(A^{\prime}, a^{\prime}\right)$, and let $\alpha_{A, A^{\prime}}$ denote the image of $E\left(A, A^{\prime}\right)$ in $\Phi_{M}$. Then the set $\left\{\alpha_{A A^{\prime}} \mid A \neq A^{\prime}\right\}$ is a set of generators for $\Phi_{M}$.

Proof. Let us note first that the statement is proved for $(G, M, R)$ as soon as it is proved for $\left(G, M, R / \operatorname{gcd}\left(r_{1}, \ldots, r_{v}\right)\right)$. We will thus assume now that $\operatorname{gcd}\left(r_{1}\right.$, $\left.\ldots, r_{v}\right)=1$. Fix a vertex $A$, and consider the subgroup $\left(\Phi_{M}\right)_{A}$ of $\Phi_{M}$ generated by $\left\{\alpha_{A A^{\prime}} \mid\right.$ all $\left.A^{\prime} \neq A\right\}$. We claim that $a \Phi_{M} \subseteq\left(\Phi_{M}\right)_{A}$. Indeed, an element $\phi \in \Phi_{M}$ is represented by the class of a vector $\left(f_{D} \mid D \in G\right)$ such that $\sum f_{D} r(D)=0$. It follows that $a \phi=-\sum \operatorname{gcd}(a, r(D)) f_{D} \alpha_{A D}$. Since $\operatorname{gcd}\left(r_{1}, \ldots, r_{v}\right)=1, \phi$ can be expressed in terms of elements of the form $\alpha_{A A^{\prime}}$.

The following is a key relation between the $\tau_{i}$ 's:

Proposition 3.5. Let $(G, M, R)$ be an arithmetical tree. Let $(C, r)$ be a vertex of degree $d \geq 2$. Keep the notation introduced in 3.3. Then $\sum_{i=1}^{d-1} \operatorname{gcd}\left(r_{i}, r_{d}\right) \tau_{i}=0$.

Proof. Consider any two distinct vertices $(A, a)$ and $\left(A^{\prime}, a^{\prime}\right)$, and let $\alpha$ denote the image of $E\left(A, A^{\prime}\right)$ in $\Phi_{M}$. The previous lemma shows that the group $\Phi_{M}$ is generated by such elements $\alpha$.

Let $\tau:=\sum_{i=1}^{d-1} \operatorname{gcd}\left(r_{i}, r_{d}\right) \tau_{i}$. We claim that $\langle\tau, \alpha\rangle=0$ for all such elements $\alpha$. This claim, proved below, implies immediately that $\tau=0$. Indeed, recall that, $\langle\cdot, \cdot\rangle$ being perfect, the element $\tau$ is trivial if and only if $\langle\tau, \phi\rangle=0$ for all $\phi \in \Phi_{M}$.

Let us now prove our claim. Assume first that the path $\mathcal{Q}$ between $A$ and $A^{\prime}$ contains the vertices $D_{i}$ and $D_{d}$ with $i \neq d$. We use (3.2.1) to compute modulo $\mathbb{Z}$ that

$$
\begin{aligned}
& \langle\tau, \alpha\rangle= \pm \operatorname{lcm}\left(a, a^{\prime}\right) \\
& \quad \times\left(\operatorname{gcd}\left(r_{i}, r_{d}\right) \operatorname{lcm}\left(r_{i}, r_{d}\right)\left(\frac{1}{r_{i} r}+\frac{1}{r r_{d}}\right)+\sum_{j \neq i, d} \operatorname{gcd}\left(r_{j}, r_{d}\right) \operatorname{lcm}\left(r_{j}, r_{d}\right)\left(\frac{1}{r r_{d}}\right)\right),
\end{aligned}
$$

which simplifies to

$$
\langle\tau, \alpha\rangle= \pm \operatorname{lcm}\left(a, a^{\prime}\right)\left(\sum_{j=1}^{d} r_{j}\right) \frac{1}{r} .
$$


Since $\sum_{j=1}^{d} r_{j}=|C \cdot C| r$, we find that $\langle\tau, \alpha\rangle=0$. When $\mathcal{Q}$ contains $D_{i}$ and $D_{j}$ with $i, j \neq d$ and $i \neq j$, we find that modulo $\mathbb{Z}$

$$
\begin{aligned}
\langle\tau, \alpha\rangle & = \pm \operatorname{lcm}\left(a, a^{\prime}\right)\left(\operatorname{gcd}\left(r_{i}, r_{d}\right) \operatorname{lcm}\left(r_{i}, r_{d}\right) \frac{1}{r_{i} r}-\operatorname{gcd}\left(r_{j}, r_{d}\right) \operatorname{lcm}\left(r_{j}, r_{d}\right) \frac{1}{r_{j} r}\right) \\
& = \pm \operatorname{lcm}\left(a, a^{\prime}\right)\left(\frac{r_{d}}{r}-\frac{r_{d}}{r}\right)=0 .
\end{aligned}
$$

It is clear that if the path $\mathcal{Q}$ contains no vertices $D_{i}$, or if it contains exactly one vertex $D_{i}$ and does not contain the vertex $C$, then $\langle\tau, \alpha\rangle=0$. It remains to consider the case where the path $\mathcal{Q}$ contains exactly one vertex $D_{i}$ and the vertex $C$. Then $C$ is an endpoint of $\mathcal{Q}$, and thus, $r$ divides $\operatorname{lcm}\left(a, a^{\prime}\right)$. When $i \neq d$, we find that

$$
\langle\tau, \alpha\rangle= \pm \operatorname{lcm}\left(a, a^{\prime}\right) \operatorname{lcm}\left(r_{i}, r_{d}\right) \operatorname{gcd}\left(r_{i}, r_{d}\right) \frac{1}{r_{i} r}
$$

is 0 modulo $\mathbb{Z}$, and when $i=d$, we find that

$$
\langle\tau, \alpha\rangle= \pm \operatorname{lcm}\left(a, a^{\prime}\right)\left(\sum_{i=1}^{d-1} \operatorname{lcm}\left(r_{i}, r_{d}\right) \operatorname{gcd}\left(r_{i}, r_{d}\right) \frac{1}{r_{i} r}\right)
$$

is also 0 modulo $\mathbb{Z}$.

\section{Some combinatorics}

Let $(G, M, R)$ be an arithmetical graph. We introduce below a measure $\gamma_{D} g_{D}$ of how "complicated" certain subgraphs $G_{D}$ of $G$ are, and we describe $G_{D}$ in Proposition 4.3 when $\gamma_{D} g_{D}$ is as small as possible. This result is needed in the proof of Theorem 6.8. A geometric motivation for the introduction of the quantity $\gamma_{D} g_{D}$ is found in the genus formula (6.1.1).

4.1. Let $(G, M, R)$ be an arithmetical graph. Fix a vertex $\left(C_{0}, r\left(C_{0}\right)\right)$ of $G$. Assume that $C_{0}$ is linked to a vertex $(D, r(D))$ by a single edge $e$ and that, when the edge $e$ is removed from $G$, then $D$ and $C_{0}$ are not in the same connected component of the resulting graph. Let $G_{D}$ denote the connected component of $G \backslash\{e\}$ that contains $D$. Consider the minor $N_{D}$ of $M$ corresponding to the vertices in $G_{D}$. Let

$$
\gamma_{D}:=\operatorname{gcd}\left(r(A) \mid A \text { a vertex of } G_{D}\right) .
$$

Then $\gamma_{D}$ divides $r\left(C_{0}\right)$. Indeed, $\gamma_{D}$ divides the multiplicity of $D$ and of all vertices linked to $D$ except possibly that of $C_{0}$. But the relation $M R=0$ implies then that $\gamma_{D}$ divides the multiplicity of $C_{0}$. Let $R_{D}$ denote the vector $R$ restricted to the vertices of $G_{D}$. By definition, we find that $R_{D} / \gamma_{D}$ is an integer vector. 
Let $\beta(G)$ denote the first Betti number of the graph $G$. Letting $d_{G}(A)$ denote the degree of a vertex $A$ in the graph $G$, we have

$$
2 \beta(G)-2=\sum_{\text {vertices } A \text { of } G}\left(d_{G}(A)-2\right) .
$$

Associated with any arithmetical graph $(G, M, R)$ is the following integer invariant $g_{0}(G) \geq \beta(G)$ [Lorenzini 1989, Theorem 4.10], defined by the formula

$$
2 g_{0}(G)=2 \beta(G)+\sum_{\text {vertices } A \text { of } G}(r(A)-1)\left(d_{G}(A)-2\right) .
$$

Let $C_{0}$ and $D$ be as above. We now associate to the pair $\left(N_{D}, R_{D}\right)$ an integer $g_{D}$, defined so that the formula below holds:

$$
\gamma_{D} g_{D}=r\left(C_{0}\right)+r(D)+\sum_{\text {vertices } A \text { of } G_{D}} r(A)\left(d_{G_{D}}(A)-2\right) .
$$

Since $\gamma_{D}$ divides $r\left(C_{0}\right)$, the invariant $g_{D}$ is indeed an integer. We can rewrite this formula as

$$
\begin{aligned}
\gamma_{D} g_{D}=2 \beta\left(G_{D}\right)+\left(r\left(C_{0}\right)-1\right)+ & (r(D)-1) \\
& +\sum_{\text {vertices } A \text { of } G_{D}}(r(A)-1)\left(d_{G_{D}}(A)-2\right),
\end{aligned}
$$

and we find that

$$
\begin{aligned}
g_{D}=2 \beta\left(G_{D}\right)+\left(\frac{r\left(C_{0}\right)}{\gamma_{D}}-1\right) & +\left(\frac{r(D)}{\gamma_{D}}-1\right) \\
& +\sum_{\text {vertices } A \text { of } G_{D}}\left(\frac{r(A)}{\gamma_{D}}-1\right)\left(d_{G_{D}}(A)-2\right) .
\end{aligned}
$$

4.2. We will make use below of the following facts. Suppose that, on $G$, the vertices $D_{0}, D_{1}, \ldots, D_{n}$ are consecutive vertices on a terminal chain and $D_{n}$ is the terminal vertex on this chain (in other words, $D_{i}$ is linked by one edge to $D_{i+1}$ for $i=0, \ldots, n-1, d_{G}\left(D_{i}\right)=2$ for $i=1, \ldots, n-1$ and $\left.d_{G}\left(D_{n}\right)=1\right)$. Then $\operatorname{gcd}\left(r\left(D_{0}\right), r\left(D_{1}\right)\right)=r\left(D_{n}\right)$, and if $\left|D_{i} \cdot D_{i}\right|>1$ for all $i=1, \ldots, n$, then

$$
r\left(D_{0}\right)>r\left(D_{1}\right)>\cdots>r\left(D_{n}\right) .
$$

Indeed, the equality $\left|D_{n} \cdot D_{n}\right| r\left(D_{n}\right)=r\left(D_{n-1}\right)$ obtained from the relation $M R=0$ shows that $r\left(D_{n}\right)$ divides $r\left(D_{n-1}\right)$ and $r\left(D_{n}\right)<r\left(D_{n-1}\right)$ if $\left|D_{n} \cdot D_{n}\right|>1$. Suppose that, for some $i$, we have $r\left(D_{i}\right)>r\left(D_{i+1}\right)$. Then $r\left(D_{i-1}\right)>r\left(D_{i}\right)$ because $\left|D_{i} \cdot D_{i}\right| r\left(D_{i}\right)=r\left(D_{i-1}\right)+r\left(D_{i+1}\right)$ and $\left|D_{i} \cdot D_{i}\right| \geq 2$. The equality $\left|D_{i} \cdot D_{i}\right| r\left(D_{i}\right)=$ $r\left(D_{i-1}\right)+r\left(D_{i+1}\right)$ implies that $\operatorname{gcd}\left(r\left(D_{i-1}\right), r\left(D_{i}\right)\right)=\operatorname{gcd}\left(r\left(D_{i}\right), r\left(D_{i+1}\right)\right)$. 
Proposition 4.3. Let $(G, M, R)$ be an arithmetical tree containing a vertex $C_{0}$ of prime multiplicity $p$. Assume that a vertex $D$ linked to $C_{0}$ by an edge e has multiplicity divisible by $p$. Let $G_{D}$ denote the connected component of $G \backslash\{e\}$ that contains $D$. Assume in addition that $G_{D}$ does not contain any vertex $A$ of degree 1 or 2 in $G$ with $|A \cdot A|=1$. Then

$$
\gamma_{D} g_{D} \geq 2(p-1)
$$

If $\gamma_{D} g_{D}=2(p-1)$, then $\gamma_{D}=1$ and $G_{D}$ is a graph of the shape depicted below, containing one node $C$ of $G$ only, of multiplicity $p$ and degree 3 in $G$. The two terminal vertices of $G$ that belong to $G_{D}$ have multiplicity 1 .

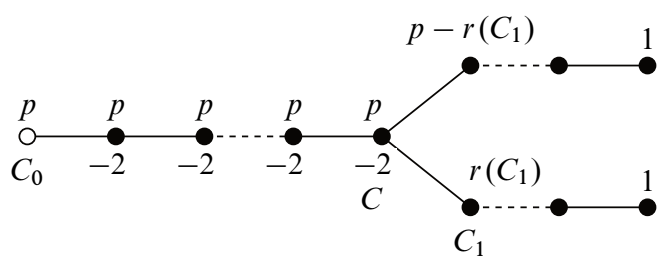

Let $\alpha$ denote the number of vertices of $G_{D}$ on the chain linking $C_{0}$ to the node $C$ of $G_{D}$ (including the node $C$ ). Let $C_{1}$ and $C_{1}^{\prime}$ denote the vertices linked to $C$ on the two terminal chains. Then $1 \leq r\left(C_{1}\right)<p$, and the minor of $M$ corresponding to the vertices of $G_{D}$ is completely determined by $p, \alpha$ and $r\left(C_{1}\right)$.

The proof of Proposition 4.3 is given in 4.6. We start with a preliminary lemma.

4.4. Let $(G, M, R)$ be an arithmetical tree. For each node $(C, r(C))$ of degree $d(C) \geq 3$ in $G$, we define an invariant $\mu(C)$ as follows. Let $\rho(C)$ denote the number of terminal chains attached to $C$, and let $D_{1}(C), \ldots, D_{\rho(C)}(C)$ be the vertices of $G$ linked to $C$ that belong each to one terminal chain attached to $C$. Let $r_{i}(C)$ denote the multiplicity of $D_{i}(C)$. The multiplicity of the terminal vertex on the chain containing $D_{i}(C)$ is $\operatorname{gcd}\left(r(C), r_{i}(C)\right)$. If no vertex $A$ on the terminal chain has $|A \cdot A|=1$, then $r_{i}(C)<r(C)$ (see 4.2). When a chain attached to $C$ is not terminal, we will call it a connecting chain. As in [Lorenzini 1989, Theorem 4.7], we let, when $\rho(C)>0$,

$$
\mu(C):=(d(C)-2)(r(C)-1)-\sum_{j=1}^{\rho(C)}\left(\operatorname{gcd}\left(r(C), r_{j}(C)\right)-1\right) .
$$

When $\rho(C)=0$, we let $\mu(C):=(d(C)-2)(r(C)-1)$. It is clear that, if $r(C)=1$, then $\mu(C)=0$.

Lemma 4.5. Assume that the terminal chains attached to $C$ do not contain a vertex $A$ with $|A \cdot A|=1$. Then $\mu(C) \geq 0$, and $\mu(C)=0$ if and only if $r(C)=1$ and $\rho(C)=0$. 
Proof. It is clear that, if a node $C$ has $\rho(C)=0$, then $\mu(C) \geq 0$, and $\mu(C)=0$ only when $r(C)=1$. Assume now that $\rho(C)>0$. Our hypothesis implies that $r(C)>\operatorname{gcd}\left(r(C), r_{i}(C)\right)$ for each vertex $D_{i}(C), i=1, \ldots, \rho(C)$. In particular, $r(C)>1$, and we need to prove that $\mu(C)>0$. Let

$$
s:=\operatorname{gcd}\left(r(C), r_{1}(C), \ldots, r_{d}(C)\right) .
$$

Assume first that $\rho(C)=d(C)$ so that $G$ has a single node. It is proven in [Lorenzini 1989, Proposition 4.1] that, if $\rho(C)=d(C)$ and $s=1$, then $\mu(C) \geq 0$. When $s>1$, define

$$
\mu_{s}(C):=(d(C)-2)\left(\frac{r(C)}{s}-1\right)-\sum_{j=1}^{\rho(C)}\left(\frac{\operatorname{gcd}\left(r(C), r_{j}(C)\right)}{s}-1\right) .
$$

The integer $\mu_{s}(C)$ is nothing but the $\mu$-invariant of the node on the arithmetical graph obtained from $G$ by dividing all its multiplicities by $s$. Thus, $\mu_{s}(C)$ is even [Lorenzini 1989, Definition 3.6] and $\mu_{s}(C) \geq 0$. Since

$$
\mu(C)=-2(s-1)+s \mu_{s}(C),
$$

we find that $\mu(C)>0$ if $\mu_{s}(C)>0$. We claim that, under our hypotheses, $\mu(C)>0$ when $s=1$. Indeed, our hypotheses imply that $r(C)>\operatorname{gcd}\left(r(C), r_{i}(C)\right)$ for each vertex $D_{i}(C), i=1, \ldots, \rho(C)$. Dropping the reference to $C$, we can write

$$
\begin{aligned}
\mu(C) & :=(d-2)(r-1)-\sum_{j=1}^{d}\left(\operatorname{gcd}\left(r, r_{i}\right)-1\right) \\
& \geq(d-2)(r-1)-d(r / 2-1)=(d-4) r / 2+2 .
\end{aligned}
$$

Thus, $\mu(C)>0$ if $d \geq 4$. Assume now that $d=3$. Then $c r=r_{1}+r_{2}+r_{3}$ for some $c$. Let $h_{i}=\operatorname{gcd}\left(r, r_{i}\right)$, and assume that $h_{1} \geq h_{2} \geq h_{3}$. Then $\left(h_{1}, h_{2}, h_{3}\right)=$ $(r / 2, r / 2, r / 2),(r / 2, r / 2, r / 3),(r / 2, r / 3, r / 3),(r / 2, r / 3, r / 4)$ cannot occur due to the divisibility $r \mid\left(r_{1}+r_{2}+r_{3}\right)$. Since the cases $\left(h_{1}, h_{2}, h_{3}\right)=(r / 3, r / 3, r / 3)$, $(r / 2, r / 4, r / 4),(r / 2, r / 3, r / 6)$ have $\mu(C)>0$, we need only consider $\left(h_{1}, h_{2}, h_{3}\right)=$ $(r / 2, r / 3, r / 5)$. In this case, $r_{1}=r / 2, r_{2}=r / 3$ or $2 r / 3$ and $r_{3}=a r / 5$ with $a=1, \ldots, 4$. The reader will check that $c r=r_{1}+r_{2}+r_{3}$ is impossible to achieve with these values, and our claim is proved.

Let us assume now that $0<\rho(C)<d(C)$. Then

$$
\begin{aligned}
\mu(C) & :=(d-2)(r-1)-\sum_{j=1}^{\rho}\left(\operatorname{gcd}\left(r, r_{i}\right)-1\right) \\
& \geq(d-2)(r-1)-(d-1)(r / 2-1)=(d-3) r / 2+1>0 .
\end{aligned}
$$

4.6. Proof of Proposition 4.3. We claim that $G_{D}$ contains a node of $G$. (This node is also a node of $G_{D}$ unless it is $D$ itself and $d_{G}(D)=3$.) Indeed, the hypotheses that $r\left(C_{0}\right) \leq r(D)$ and $|D \cdot D|>1$ imply that $d_{G}(D)>1$ because the relation $M R=0$ 
provides otherwise for the equality $|D \cdot D| r(D)=r\left(C_{0}\right)$, which is a contradiction. Suppose then that $D$ is connected in $G_{D}$ to $D_{1}$. If $d_{G}(D)=2$, then we find from the relation $|D \cdot D| r(D)=r\left(C_{0}\right)+r\left(D_{1}\right)$ that $r(D) \leq r\left(D_{1}\right)$. Repeating this discussion with $D$ and $D_{1}$ instead of $C_{0}$ and $D$, we find that the graph $G_{D}$ has a chain of increasing multiplicities $r(D) \leq r\left(D_{1}\right) \leq \cdots$, which eventually leads to a node of $G_{D}$ (and of $G$ ).

In $G, C_{0}$ and $D$ are adjacent vertices. Consider the connected component $\mathcal{G}$ of $G \backslash\{D\}$ that contains $C_{0}$. Two cases can occur: either (a) $\mathcal{G}$ contains a node of $G$, or (b) $\mathcal{G}$ does not contain a node of $G$, in which case we will call $\mathcal{G}$ a terminal chain of $G$. In the latter case, the terminal vertex on this chain has multiplicity $\operatorname{gcd}\left(r\left(C_{0}\right), r(D)\right)$ (see 4.2), which equals $r\left(C_{0}\right)$ by hypothesis. The definition of $\gamma_{D} g_{D}$ in (4.1.2), along with the fact that we assume that $G$ is a tree, allow us to write

$$
\gamma_{D} g_{D}=\left(r\left(C_{0}\right)-1\right)+\sum_{\text {vertices } A \text { of } G_{D}}(r(A)-1)\left(d_{G}(A)-2\right)
$$

In case (a), $C_{0}$ is not on a terminal chain of $G$ so that, by definition of $\mu(C)$ in 4.4 , we can write

$$
\gamma_{D} g_{D}=\left(r\left(C_{0}\right)-1\right)+\sum_{\text {nodes } C \text { of } G \text { in } G_{D}} \mu(C)
$$

(where $\mu(C)$ is computed viewing $C$ as a node of $G$ and not of $G_{D}$ ). In case (b) where $C_{0}$ is on a terminal chain of $G$ whose terminal vertex has multiplicity $r\left(C_{0}\right)$, we have

$$
\gamma_{D} g_{D}=2\left(r\left(C_{0}\right)-1\right)+\sum_{\operatorname{nodes} C \text { of } G \text { in } G_{D}} \mu(C) .
$$

We prove below case (a). The arguments to prove (b) are similar and are left to the reader. Case (b) will not be used in the remainder of this article.

Assume that we are in case (a). We can apply Lemma 4.5, and we obtain that each term $\mu(C)$ in the above sum is nonnegative. In view of (4.6.1), since $r\left(C_{0}\right)=p$ by hypothesis, we need to show that $\sum_{\text {nodes } C} \mu(C) \geq p-1$, and we need to describe the graphs for which $\sum_{\text {nodes } C} \mu(C)=(p-1)$.

Denote by $C$ the node of $G$ closest to $C_{0}$ in $G_{D}$. (This node could be $D$.) The multiplicity of $C$ is divisible by $p$ since $p$ divides the consecutive multiplicities $r\left(C_{0}\right)$ and $r(D)$ (similar argument as in 4.2). Let $n p$ denote the multiplicity of $C$.

Suppose that $C$ (of degree $d$ in $G$ ) has only one connecting chain. If $n=1$, then all terminal multiplicities at $C$ equal 1 and $\mu(C)=(d-2)(p-1)$. The case $d=3$ leads to the case described in the statement of Proposition 4.3 with $\mu(C)=(p-1)$, $\gamma_{D} g_{D}=2(p-1)$ and $\gamma_{D}=1$. When $d>3$, we have $\mu(C)>p-1$, as desired. 
When $n>1$, the inequality

$$
\begin{aligned}
\mu(C) & \geq(d-2)(n p-1)-(d-1)(n p / 2-1) \\
& =(d-2) n p / 2-n p / 2+1
\end{aligned}
$$

shows that we have $\mu(C)>p-1$ unless $d=3$. When $n>1$ and $d=3$, every vertex on the chain linking $C$ to $C_{0}$ has multiplicity divisible by $p$. Thus, either (i) both terminal multiplicities of $C$ are coprime to $p$ (call them $n_{1}$ and $n_{2}$ ), or (ii) both are divisible by $p$ (call them $m_{1} p$ and $m_{2} p$ ).

In case (i), $\mu(C)=n p-n_{1}-n_{2}+1$ with $n_{1}$ and $n_{2}$ dividing $n$. It follows that $\mu(C) \geq n(p-2)+1$. Clearly, $\mu(C)>p-1$ unless $p=2$. Assume that $p=2$. If $\left(n_{1}, n_{2}\right) \neq(n, n)$, we find that $\mu(C)=n(p-1)+1>(p-1)$. The case $\left(n_{1}, n_{2}\right)=(n, n)$ cannot happen because, in that case, $n$ divides the multiplicity of all the components linked to $C$, which implies then that $n=2$. But a node of multiplicity 4 cannot have exactly three vertices of multiplicity 2 attached to it.

In case (ii), $\mu(C)=\left(n-m_{1}-m_{2}\right) p+1$ with $m_{1}$ and $m_{2}$ dividing $n$. The equality $\left(n-m_{1}-m_{2}\right)=0$ is not possible. Indeed, it is only possible if $m_{1}=m_{2}=n / 2$. But since $\operatorname{gcd}\left(m_{1}, m_{2}\right)=1$, this case can happen only if $n=2$. But then $|C \cdot C|$ would equal $3 / 2$, a contradiction. It follows that $\mu(C)=\left(n-m_{1}-m_{2}\right) p+1>p-1$.

Suppose now that $C$, of multiplicity $n p$, has at least two connecting chains. If $n>1$, then

$$
\mu(C) \geq(d-2)(n p-1)-(d-2)(n p / 2-1)=(d-2) n p / 2>p-1,
$$

as desired. If $n=1$, then $\mu(C)=(d-2)(p-1)$. Thus, $\mu(C)>p-1$ if $d>3$. Suppose now that $d=3$. Since $G_{D}$ is a tree with a node $C$ of degree $3, G_{D}$ must have at least three terminal vertices. Thus, there must exist at least one additional node $C^{\prime}$ on the graph $G_{D}$ that has a terminal chain. It follows that $\mu\left(C^{\prime}\right) \geq 1$ (Lemma 4.5) and, therefore, $\mu(C)+\mu\left(C^{\prime}\right)>p-1$, as desired.

4.7. To conclude the proof of Proposition 4.3, we now specify the intersection matrix in the case where $\gamma_{D} g_{D}=2(p-1)$. Let $(G, M, R)$ be as in Proposition 4.3, and assume that the vertex $D$ is such that $\gamma_{D} g_{D}=2(p-1)$. Let $N_{D}$ denote the matrix $M$ restricted to the vertices of $G_{D}$. Let $\alpha$ denote the number of vertices of $G_{D}$ on the chain linking $C_{0}$ to the node $C$ of $G_{D}$ (including the node $C$ ). Each of these vertices except $C$ is of degree 2. The multiplicity of $C$ is $p$. Since we assume that no vertex of degree 2 has self-intersection -1 , we find that the multiplicity of each of these vertices must be $p$. It follows that each of these vertices except possibly $C$ must have self-intersection -2 .

Let $C_{1}$ and $C_{1}^{\prime}$ denote the vertices linked to $C$ on the two terminal chains. Since they have degree 1 or 2 and cannot have self-intersection -1 , we find that $1 \leq r\left(C_{1}\right)<r(C)=p$ and $r\left(C_{1}^{\prime}\right)<r(C)$. Moreover, from $M R=0$, we find 
that $p+r\left(C_{1}\right)+r\left(C_{1}^{\prime}\right)=p|C \cdot C|$. It follows that $|C \cdot C|=2$, and $r\left(C_{1}^{\prime}\right)=$ $p-r\left(C_{1}\right)$. We claim that $N_{D}$ depends only on $p, \alpha$ and $r\left(C_{1}\right)$, and we write it as $N_{D}=N\left(p, \alpha, r\left(C_{1}\right)\right)$. Indeed, the pair $\left(p, r\left(C_{1}\right)\right)$ completely determines all multiplicities and all self-intersections on the terminal chain containing $C_{1}$ : use $(r, s)=\left(p, r\left(C_{1}\right)\right)$ in 4.8 below to determine the self-intersections and multiplicities of the terminal chain. Similarly, the pair $\left(p, r\left(C_{1}^{\prime}\right)\right)$ completely determines all multiplicities and all self-intersections on the terminal chain containing $C_{1}^{\prime}$. This conclude the proof of Proposition 4.3. The matrix $N_{D}$ is an intersection matrix also introduced in [Lorenzini 2013a, Example 3.18].

4.8. Recall the following standard construction. Given an ordered pair of positive integers $r>s$ with $\operatorname{gcd}(r, s)=1$, we construct an associated intersection matrix $N=N(r, s)$ with vector $R=R(r, s)$ and $N R=-r e_{1}$ as follows (where $e_{1}$ denotes the first standard basis vector of $\left.\mathbb{Z}^{n}\right)$. Using the division algorithm, we can find positive integers $b_{1}, \ldots, b_{m}$ and $s_{1}=s>s_{2}>\cdots>s_{m}=1$ such that $r=b_{1} s-s_{2}$, $s_{1}=b_{2} s_{2}-s_{3}$ and so on until we get $s_{m-1}=b_{m} s_{m}$. These equations are best written in matrix form:

$$
\left(\begin{array}{cccc}
-b_{1} & 1 & \ldots & 0 \\
1 & -b_{2} & \ddots & \\
& \ddots & \ddots & 1 \\
0 & \ldots & 1 & -b_{m}
\end{array}\right)\left(\begin{array}{c}
s_{1} \\
\vdots \\
\vdots \\
s_{m}
\end{array}\right)=\left(\begin{array}{c}
-r \\
0 \\
\vdots \\
0
\end{array}\right)
$$

We let $N(r, s)$ denote the above square matrix and $R(r, s)$ be the column matrix on the left of the "equals" sign. It is well-known that $\operatorname{det}(N(r, s))= \pm r$ (see [Lorenzini 2000, Lemma 2.6]). We recall also for use in Corollary 6.12 that

$$
\frac{1}{r s}+\frac{1}{s s_{2}}+\cdots+\frac{1}{s_{m-1} s_{m}}=\frac{c}{r}
$$

where $0<c<r$ is such that $r \mid c s-1$ (see [Lorenzini 2000, Lemmas 2.8 and 2.6]).

Remark 4.9. In Proposition 4.3, the hypothesis that $\gamma_{D} g_{D}=2(p-1)$ allowed us to completely describe the graph $G_{D}$. For a fixed $\gamma_{D} g_{D}>2(p-1)$, the situation is much more complicated and several possible types of graphs $G_{D}$ may occur. It would follow from our guess in 6.2 that, for applications to models of curves, it suffices to classify the cases where $\gamma_{D} g_{D}$ is a multiple of $p-1$. We give below several possible types of graphs $G_{D}$ with $\gamma_{D} g_{D}=3(p-1)$ when $p$ is odd.

(a) $G_{D}$ is a graph with one node of $G$ only, of multiplicity $p$ and degree 4 in $G$. The three terminal vertices of $G$ that belong to $G_{D}$ have multiplicity 1 . 


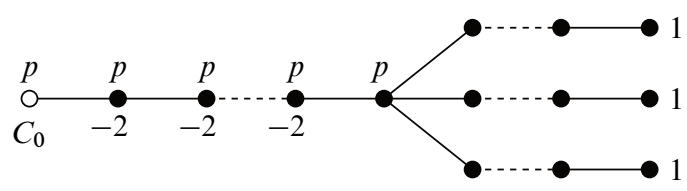

To completely determine the intersection matrix $N_{D}$ and the vector $R_{D}$, one needs to also provide the multiplicities $r_{1}, r_{2}$ and $r_{3}$, of the first vertices on each of the three terminal chains, with the conditions $1 \leq r_{1}, r_{2}, r_{3}<p$ and $r_{1}+r_{2}+r_{3}$ divisible by $p$. Such data can only be provided when $p$ is odd. The self-intersection of the node is then $-\left(p+r_{1}+r_{2}+r_{3}\right) / p=-2$ or -3 .

(b) $G_{D}$ is a graph with one node of $G$ only, of multiplicity $2 p$ and degree 3 in $G$. The two terminal vertices of $G$ that belong to $G_{D}$ have multiplicity 1 and 2, respectively.

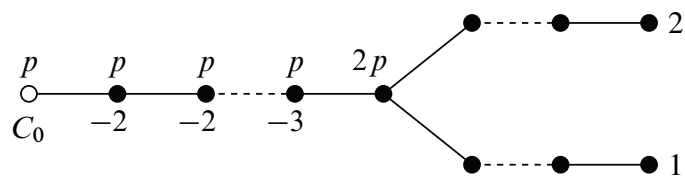

(c) $G_{D}$ is a graph with 2 nodes $C$ and $C^{\prime}$ of $G$. Let $C$ be the node closest to $C_{0}$ in $G_{D}$. It has multiplicity $p$ and degree 3 in $G$, and it has a single terminal chain with terminal multiplicity 1 . The node $C^{\prime}$ is connected to $C$ by a connecting chain that contains a vertex of multiplicity coprime to $p$.

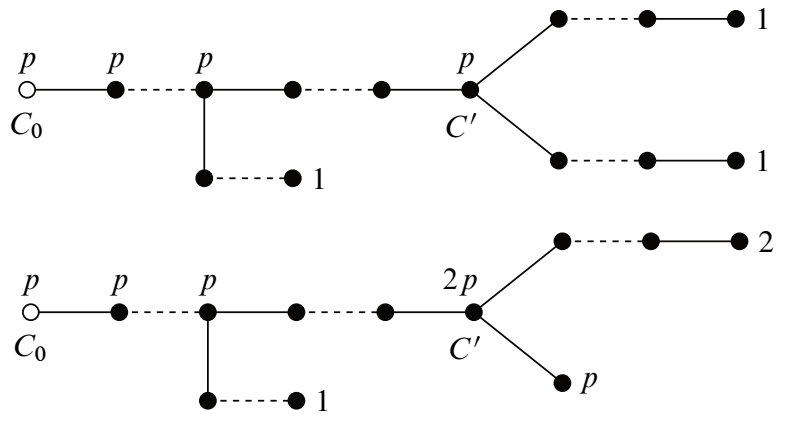

We conclude this section with some general remarks concerning the invariant $g_{D}$ introduced in (4.1.2).

Remark 4.10. Let $(G, M, R)$ be an arithmetical graph. As at the beginning of this section, fix a vertex $\left(C_{0}, r\left(C_{0}\right)\right)$ of $G$. Assume that $C_{0}$ is linked to a vertex $(D, r(D))$ by a single edge $e$ and that, when the edge $e$ is removed from $G$, then $D$ and $C_{0}$ are not in the same connected component of the resulting graph. Let $G_{D}$ denote the connected component of $G \backslash\{e\}$ that contains $D$. Consider the minor $N=N_{D}$ of $M$ corresponding to the vertices in $G_{D}$. Let $n$ denote the number of vertices of $G_{D}$. 
(a) The integer $g_{D}$ depends only on the matrix $N_{D}$ and the vertex $D$ on the graph $G_{D}$. To prove this statement, we show that the vector $R_{D} / \gamma_{D}$ is completely determined by $N_{D}$ and the vertex $D$. Indeed, let us number the vertices of $G_{D}$ such that $D$ is the first vertex numbered. Then $R_{D} / \gamma_{D}$ is a vector with positive coefficients such that $N_{D}\left(R_{D} / \gamma_{D}\right)={ }^{t}\left(-r\left(C_{0}\right) / \gamma_{D}, 0, \ldots, 0\right)$ (where the superscript $t$ indicates the transpose vector). The existence of such a relation insures that $N_{D}$ is negativedefinite (see [Lorenzini 2013a, §3.3]), and the vector $R_{D} / \gamma_{D}$ is a rational multiple of the first column of the unique matrix $N^{*}$ such that $N N^{*}=N^{*} N=\operatorname{det}(N) \operatorname{Id}_{n}$ [Lorenzini 2013a, Definition 3.4]. The integer $r\left(C_{0}\right) / \gamma_{D}$ is the order in $\mathbb{Z}^{n} / \operatorname{Im}(N)$ of the class of the first basis vector $e_{1}$ [Lorenzini 2013a, Lemma 3.5].

(b) The integer $g_{D}$ is nonnegative. More precisely,

$$
g_{D}-2 \beta\left(G_{D}\right) \geq\left(\frac{r\left(C_{0}\right)}{\gamma_{D}}\right)+\operatorname{gcd}\left(\frac{r(D)}{\gamma_{D}}, \frac{r\left(C_{0}\right)}{\gamma_{D}}\right)-2 \geq 0 .
$$

To prove the first inequality, complete the pair $\left(N, R_{D} / \gamma_{D}\right)$ into an arithmetical graph $\left(G^{\prime}, M^{\prime}, R^{\prime}\right)$ by adding a chain attached to $D$, as in [Lorenzini 2013a, §3.15]. Clearly, $\beta\left(G^{\prime}\right)=\beta\left(G_{D}\right)$. The graphs $G^{\prime}$ and $G_{D}$ differ in only two vertices of degree not equal to 2: the terminal vertex on the new terminal chain on $G^{\prime}$ has terminal multiplicity $\operatorname{gcd}\left(r(D) / \gamma_{D}, r\left(C_{0}\right) / \gamma_{D}\right)$, and $d_{G^{\prime}}(D)=d_{G_{D}}(D)+1$. Using (4.1.1) and (4.1.3), it is easy to show that

$$
\begin{aligned}
2 g_{0}\left(G^{\prime},\right. & \left.M^{\prime}, R^{\prime}\right)-2 \beta\left(G^{\prime}\right) \\
& =g_{D}-2 \beta\left(G_{D}\right)-\left(\frac{r\left(C_{0}\right)}{\gamma_{D}}-1\right)-\left(\operatorname{gcd}\left(\frac{r(D)}{\gamma_{D}}, \frac{r\left(C_{0}\right)}{\gamma_{D}}\right)-1\right) .
\end{aligned}
$$

The integer $g_{0}\left(G^{\prime}\right)-\beta\left(G^{\prime}\right)$ is always nonnegative [Lorenzini 1989, Theorem 4.10], and the statement follows.

(c) In analogy with the arithmetic genus of curves on surfaces, we define, given $Z \in \mathbb{Z}^{n}$, a (possibly negative) integer $p_{a}(Z)$ as follows. If $Z=C_{i}$ is a vertex of $G_{D}$, we let $p_{a}(Z)=0$. We let $p_{a}\left(r C_{i}\right)$ be defined by the formula $2 p_{a}\left(r C_{i}\right)-2=$ $r^{2} C_{i}^{2}+r\left(\left|C_{i}^{2}\right|-2\right.$ ) (where we have abbreviated $C_{i} \cdot C_{i}$ by $C_{i}^{2}$ ). Since $r^{2}-r$ is always even, $p_{a}\left(r C_{i}\right)$ is an integer. In general, when $Z=\sum_{i=1}^{n} r_{i} C_{i}$, we let

$$
Z^{2}:=\sum_{1 \leq i, j \leq n} r_{i} r_{j}\left(C_{i} \cdot C_{j}\right)
$$

and set

We leave it to the reader to check that

$$
2 p_{a}(Z)-2:=Z^{2}+\sum_{i=1}^{n} r_{i}\left(\left|C_{i}^{2}\right|-2\right) .
$$

$$
g_{D}=2 p_{a}\left(R_{D} / \gamma_{D}\right)-2+\frac{r(D)}{\gamma_{D}}\left(\frac{r\left(C_{0}\right)}{\gamma_{D}}+1\right)
$$


(d) The integer $g_{D}$ is even when either $r\left(C_{0}\right)$ is odd or $r(D)$ is even. This can be seen from the formula for $g_{D}$ in (c) or from (4.10.2).

(e) Assume that $G_{D}$ is a tree. Then the order $|\operatorname{det}(N)|$ of the group $\Phi_{N}:=\mathbb{Z}^{n} / N\left(\mathbb{Z}^{n}\right)$ can be computed completely in terms of the vector $R_{D} / \gamma_{D}$ and of the graph $G_{D}$ (see [Lorenzini 2013a, Theorem 3.14]), and we find that

$$
|\operatorname{det}(N)|=\frac{r(D)}{\gamma_{D}} \frac{r\left(C_{0}\right)}{\gamma_{D}} \prod_{\text {vertices } A \text { of } G_{D}}\left(\frac{r(A)}{\gamma_{D}}\right)^{d_{G_{D}}(A)-2},
$$

where $d_{G_{D}}(A)$ is the degree of the vertex $A$ in the graph $G_{D}$. Recall now the formula (4.1.3):

$$
g_{D}=\left(\frac{r(D)}{\gamma_{D}}-1\right)+\left(\frac{r\left(C_{0}\right)}{\gamma_{D}}-1\right)+\sum_{\text {vertices } A \text { of } G_{D}}\left(\frac{r(A)}{\gamma_{D}}-1\right)\left(d_{G_{D}}(A)-2\right) .
$$

This last expression is surprisingly similar to the expression for $|\operatorname{det}(N)|$. This motivates the following result.

Let $x>0$ be any integer, and define the function $\ell(x):=\sum_{q \text { prime }} \operatorname{ord}_{q}(x)(q-1)$. Then

$$
\ell(|\operatorname{det}(N)|) \leq g_{D} .
$$

This result is not used in the remainder of this paper, and we will provide here only a sketch of proof.

Sketch of proof. We complete the pair $\left(N, R_{D} / \gamma_{D}\right)$ into an arithmetical graph $\left(G^{\prime}, M^{\prime}, R^{\prime}\right)$ by adding a chain attached to $D$, as in [Lorenzini 2013a, §3.15]. The order of the component group $\Phi\left(M^{\prime}\right)$ is given in [Lorenzini 1989, Corollary 2.5], and the relation between $\operatorname{det}(N)$ and $\left|\Phi\left(M^{\prime}\right)\right|$ is discussed in the proof of Theorem 3.14 in [Lorenzini 2013a]. We can then bound $\left|\Phi\left(M^{\prime}\right)\right|$ in terms of $g_{0}\left(G^{\prime}, M^{\prime}, R^{\prime}\right)$ using [Lorenzini 1989, Corollary 4.8], which states that $\ell\left(\left|\Phi\left(M^{\prime}\right)\right|\right) \leq 2 g_{0}\left(G^{\prime}, M^{\prime}, R^{\prime}\right)$. The inequality $\ell(|\operatorname{det}(N)|) \leq g_{D}$ follows then from (4.10.2).

\section{The quotient construction}

Let $K$ be a complete discrete valuation field with valuation $v$, ring of integers $\mathcal{O}_{K}$, uniformizer $\pi_{K}$ and residue field $k$ of characteristic $p>0$, assumed to be algebraically closed. Let $X / K$ be a smooth proper geometrically connected curve of genus $g>0$. When $g=1$, assume in addition that $X(K) \neq \varnothing$. Assume that $X / K$ does not have semistable reduction over $\mathcal{O}_{K}$ and that it achieves good reduction after a cyclic extension $L / K$ of prime degree $q$.

Let $H$ denote the Galois group of $L / K$. Let $\mathcal{Y} / \mathcal{O}_{L}$ be the smooth model of $X_{L} / L$. Let $\sigma$ denote a generator of $H$. By minimality of the model $\mathcal{Y}, \sigma$ defines an automorphism of $\mathcal{Y}$ also denoted by $\sigma$ (but note that $\sigma: \mathcal{Y} \rightarrow \mathcal{Y}$ is not a morphism 
of $\mathcal{O}_{L}$-schemes). We also denote by $\sigma$ the automorphism of $\mathcal{Y}_{k}$ induced by the action of $\sigma$ on $\mathcal{Y}$. Let $\mathcal{Z} / \mathcal{O}_{K}$ denote the quotient $\mathcal{Y} / H$, and let $\alpha: \mathcal{Y} \rightarrow \mathcal{Z}$ denote the quotient map. The scheme $\mathcal{Z}$ is normal. The map $\alpha$ induces a natural map $\mathcal{Y}_{k} \rightarrow \mathcal{Z}_{k}^{\text {red }}$ that factors as follows:

$$
\mathcal{Y}_{k} \stackrel{\rho}{\rightarrow} \mathcal{Y}_{k} /\langle\sigma\rangle \rightarrow \mathcal{Z}_{k}^{\text {red }}
$$

5.1. We claim that the first map is Galois of order $|H|$ and that the second map is the normalization map of $\mathcal{Z}_{k}^{\text {red }}$. Indeed, let $\operatorname{Spec}(B)$ denote a dense open set of $\mathcal{Y}$ invariant under the action of $H$. Then $\operatorname{Spec}\left(B^{H}\right)$ is a dense open set of $\mathcal{Z}$. Let $A:=B^{H}$. Let $P_{B}=\left(\pi_{L}\right)$ denote the prime ideal of $B$ corresponding to $\mathcal{Y}_{k}$, and let $P_{A}:=P_{B} \cap A$. We have the natural maps

$$
B^{H} / P_{A} \hookrightarrow\left(B / P_{B}\right)^{H} \hookrightarrow B / P_{B} .
$$

The extension of discrete valuation rings $\left(B^{H}\right)_{P_{A}} \rightarrow B_{P_{B}}$ induces an extension of residue fields $\left(B^{H}\right)_{P_{A}} / P_{A}\left(B^{H}\right)_{P_{A}} \rightarrow B_{P_{B}} / P_{B} B_{P_{B}}$. We claim that this extension has degree $|H|$. Indeed, our assumption is that the curve $X / K$ does not have good reduction over $\mathcal{O}_{K}$. If the residue extension is trivial, the normalization of the curve $\mathcal{Z}_{k}^{\text {red }}$ is isomorphic to $\mathcal{Y}_{k}$ and, thus, is of genus $g$. In addition, we find that $P_{A} B_{P_{B}}=\left(P_{B} B_{P_{B}}\right)^{|H|}$ so that $\pi_{K} A_{P_{A}}=\left(P_{A} A_{P_{A}}\right)$. The special fiber of $\mathcal{Z}$ is then reduced, and the curve $X / K$ has good reduction over $\mathcal{O}_{K}$, a contradiction. It follows then that $P_{A} B_{P B}=P_{B} B_{P B}$ so that $\pi_{K} A_{P_{A}}=\left(P_{A} A_{P_{A}}\right)^{|H|}$. Hence, the multiplicity in $\mathcal{Z}$ of the irreducible component $\mathcal{Z}_{k}^{\text {red }}$ equals $|H|$.

It is easy to check that, for any $x \in\left(B / P_{B}\right)^{H},|H| x$ and $x^{|H|}$ belong to $A / P_{A}$. Thus, when $|H| \neq p, A / P_{A}$ and $\left(B / P_{B}\right)^{H}$ have the same field of fractions. When $|H|=p$, it could happen that $A / P_{A}$ and $\left(B / P_{B}\right)^{H}$ do not have the same field of fractions, and then the extension of fields of fractions is purely inseparable of degree $p$ with $\left(B / P_{B}\right)^{H}=B / P_{B}$. It follows that the special fiber of $\mathcal{Z}$ also has genus $g$. When $g>1$, this is not possible since the multiplicity of $\mathcal{Z}_{k}$ is $p$. When $g=1$, it could happen that $\mathcal{Z}$ is the minimal model of $X / K$ with a multiple special fiber. This case cannot happen in our situation because we assumed $X(K) \neq \varnothing$ : a $K$-rational point always reduces to a smooth point in the special fiber. Thus, the automorphism $\sigma: \mathcal{Y}_{k} \rightarrow \mathcal{Y}_{k}$ is not trivial. We find that $A / P_{A}$ and $\left(B / P_{B}\right)^{H}$ have the same fields of fractions so that the Dedekind domain $\left(B / P_{B}\right)^{H}$ is the integral closure of $A / P_{A}$.

5.2. Let $P_{1}, \ldots, P_{d}$ be the ramification points of the map $\mathcal{Y}_{k} \rightarrow \mathcal{Y}_{k} /\langle\sigma\rangle$. Let $Q_{1}, \ldots, Q_{d}$ be their images in $\mathcal{Z}$. The normal scheme $\mathcal{Z}$ is singular exactly at $Q_{1}, \ldots, Q_{d}$. Indeed, the morphism $\mathcal{Y} \rightarrow \mathcal{Z}$ is unramified outside these points. If the point $Q_{i}$ were regular, the morphism would be flat above $Q_{i}$ [Altman and Kleiman 1970, Corollary V.3.6] and the branch locus would then be pure of codimension 1 [Altman and Kleiman 1970, Theorem VI.6.8], a contradiction. 
Consider the regular model $\mathcal{X} \rightarrow \mathcal{Z}$ obtained from $\mathcal{Z}$ by a minimal desingularization. After finitely many blow-ups $\mathcal{X}^{\prime} \rightarrow \mathcal{X}$, we can assume that the model $\mathcal{X}^{\prime}$ is such that $\mathcal{X}_{k}^{\prime}$ has smooth components and normal crossings and is minimal with this property. Let $f$ denote the composition $\mathcal{X}^{\prime} \rightarrow \mathcal{Z}$. Let $C_{0} / k$ denote the strict transform in $\mathcal{X}^{\prime}$ of the irreducible closed subscheme $\mathcal{Z}_{k}^{\text {red }}$ of $\mathcal{Z}$. The curve $C_{0}$ has multiplicity $|H|$ in $\mathcal{X}^{\prime}$. Let $D_{1}, \ldots, D_{d}$ denote the irreducible components of $\mathcal{X}_{k}^{\prime}$ that meet $C_{0}$. Let $r_{i}$ denote the multiplicity of $D_{i}, i=1, \ldots, d$. We assume $d \geq 1$. Our main theorem in this section is this:

Theorem 5.3. Let $X / K$ be a smooth proper geometrically connected curve of genus $g>0$ with $X(K) \neq \varnothing$ if $g=1$. Assume that $X / K$ does not have semistable reduction over $\mathcal{O}_{K}$ and that it achieves good reduction after a cyclic extension $L / K$ with Galois group $H$ of prime degree $p$. Keep the above notation, and let $Q_{i}$ be a singular point of the quotient $\mathcal{Z}:=\mathcal{Y} / H$. Let $G_{Q_{i}}$ denote the graph associated with the curve $f^{-1}\left(Q_{i}\right)$. Let $G$ denote the graph associated with the special fiber $\mathcal{X}_{k}^{\prime}$. Then, for all $i=1, \ldots, d$, the graph $G_{Q_{i}}$ contains a node of $G$ and $p$ divides $r_{i}$.

Proof. When $d=1$, the theorem is immediate: the component $C_{0}$ of multiplicity $p$ is a terminal vertex of the graph of $\mathcal{X}^{\prime}$, and thus, $p\left|C_{0} \cdot C_{0}\right|=r_{1}$. Assume that $G_{Q_{1}}$ does not contain a node of $G$. Then since $d=1, G$ does not contain a node. Since the resolution is minimal with normal crossings, none of the components of $\mathcal{X}_{k}^{\prime}$ can have self-intersection -1 except possibly for $C_{0}$. It is clear that the graph $G$ is not reduced to a single vertex since the model $\mathcal{Z}$ is singular. Thus, the graph $G$ has a second terminal vertex $C^{\prime}$ in addition to $C_{0}$. But then, walking on $G$ from $C^{\prime}$ towards $C_{0}$, we find that the multiplicities can only be strictly increasing. This is a contradiction since all multiplicities on $G$ are divisible by $p$ (because two consecutive ones are), and $G$ must contain a node. We assume from now on that $d>1$.

Let $A:=\operatorname{Jac}(X / K)$. Let $\mathcal{A}_{K} / \mathcal{O}_{K}$ denote the Néron model of $A / K$. Let $\mathcal{A}_{L} / \mathcal{O}_{L}$ denote the Néron model of $A_{L} / L$, and denote by $\eta: \mathcal{A}_{K} \times{ }_{\mathcal{O}_{K}} \mathcal{O}_{L} \rightarrow \mathcal{A}_{L}$ the canonical map induced by the functoriality property of Néron models. The special fiber $\left(\mathcal{A}_{K}\right)_{k}$ is an extension of a finite group $\Phi_{A / K}$, called the group of components, by the connected component of zero $\left(\mathcal{A}_{K}\right)_{k}^{0}$ of $\left(\mathcal{A}_{K}\right)_{k}$ :

$$
0 \rightarrow\left(\mathcal{A}_{K}\right)_{k}^{0} \rightarrow\left(\mathcal{A}_{K}\right)_{k} \rightarrow \Phi_{A / K} \rightarrow 0 .
$$

Assume by contradiction that $p$ is coprime to one of the $r_{i}$ 's. Without loss of generality, we may assume that $p \nmid r_{d}$. For each $i=1, \ldots, d$, choose a point $x_{i} \in D_{i}$ such that $x_{i}$ is a regular point of $\left(\mathcal{X}_{k}^{\prime}\right)^{\text {red }}$. Since $K$ is complete, we can find a closed point $R_{i}$ of $X$ of degree $r_{i}$ over $K$ and such that the closure of $R_{i}$ in $\mathcal{X}^{\prime}$ meets the special fiber $\mathcal{X}_{k}$ exactly in $x_{i}$ (see, e.g., [Gabber et al. 2013, Proposition 8.4(3)]). For each $i=1, \ldots, d-1$, consider the following divisor of degree 0 on $X$ : 


$$
S_{i}:=\frac{r_{d}}{\operatorname{gcd}\left(r_{i}, r_{d}\right)} R_{i}-\frac{r_{i}}{\operatorname{gcd}\left(r_{i}, r_{d}\right)} R_{d}
$$

We also denote by $S_{i}$ its image in $\operatorname{Jac}(X) / K$. We recall below Raynaud's description of the Néron model of a Jacobian in order to be able to describe explicitly the image of $S_{i}$ under both the reduction map $\operatorname{Jac}(X)(K) \rightarrow \Phi_{A / K}$ and the reduction map $\operatorname{Jac}(X)(L) \rightarrow\left(\mathcal{A}_{L}\right)_{k}(k)$. We will be able to contradict the hypothesis that $p \nmid r_{d}$ by considering the reductions of $\sum_{i=1}^{d-1} \operatorname{gcd}\left(r_{i}, r_{d}\right) S_{i}$.

Raynaud [1970] exhibited an explicit separated quotient $Q_{K} / \mathcal{O}_{K}$ of the open subfunctor of $\operatorname{Pic}_{\mathcal{X}^{\prime}} / \mathcal{O}_{K}$ consisting of line bundles of total degree 0 , and he showed that, when the residue field $k$ is algebraically closed, $Q_{K} / \mathcal{O}_{K}$ is isomorphic to the Néron model of $A / K$ [Bosch et al. 1990, Theorem 9.5.4(a)]. The canonical map $Q_{K}(K) \rightarrow \Phi_{Q_{K}}$ is described as follows [Bosch et al. 1990, Lemma 9.5.9, Theorem 9.6.1]. Represent an element of $Q_{K}(K)$ by a line bundle $\mathcal{L}$ on $X$ of degree 0 . Let $\overline{\mathcal{L}}$ denote an extension of $\mathcal{L}$ to $\mathcal{X}^{\prime}$. Number the irreducible components of $\mathcal{X}_{k}^{\prime}$ as $C_{1}, \ldots, C_{v}$. Consider the map $\bigoplus_{i} \mathbb{Z} C_{i} \rightarrow \operatorname{Hom}\left(\bigoplus_{i} \mathbb{Z} C_{i}, \mathbb{Z}\right)$ that sends $C_{i}$ to the map $\delta_{C_{i}}$ with $\delta_{C_{i}}\left(C_{j}\right):=\left(C_{i} \cdot C_{j}\right)$. The group $\Phi_{M}$ is isomorphic to the torsion subgroup of the cokernel of this map. The group of components $\Phi_{Q_{K}}$ is isomorphic to $\Phi_{M}$, and under this isomorphism, the image of $\mathcal{L}$ under $Q_{K}(K) \rightarrow \Phi_{Q_{K}}$ is the map $\delta_{\mathcal{L}}$ with $\delta_{\mathcal{L}}\left(C_{i}\right):=\left(C_{i} \cdot \overline{\mathcal{L}}\right)$. It follows immediately from these facts that the image in $\Phi_{Q_{K}}$ of $S_{i} \in \operatorname{Jac}(X)(K)$ can be identified with the image $\tau_{i}$ of the vector $E\left(D_{i}, D_{d}\right)$ in $\Phi_{M}$ (notation as in 3.1 and 3.3).

Consider now the reduction map $Q_{L}(L) \rightarrow\left(Q_{L}\right)_{k}(k)$. The closure of any point in the preimage under $X_{L} \rightarrow X$ of the closed point $R_{i}$ meets the special fiber of the smooth model $\mathcal{Y}$ of $X_{L}$ only at the point $P_{i}$. The line bundle $\mathcal{L}$ corresponding to the divisor $S_{i}$ pulls back to a line bundle $\mathcal{L}_{L}$ on $X_{L}$. We find that the reduction of $\mathcal{L}_{L} \in \operatorname{Jac}\left(X_{L}\right)(L)$ is the point of $\operatorname{Jac}\left(\mathcal{Y}_{k}\right)(k)$ corresponding to the divisor $\operatorname{lcm}\left(r_{i}, r_{d}\right)\left(P_{i}-P_{d}\right)$.

We may now find a contradiction to the assertion that $p \nmid r_{d}$ when the quotient of $\mathcal{Y}_{k}$ by the action of $H$ has genus 0 . As we indicated above, the element $\sum_{i=1}^{d-1} \operatorname{gcd}\left(r_{i}, r_{d}\right) S_{i}$ in $\operatorname{Jac}(X)(K)$ reduces to the element $\sum_{i=1}^{d-1} \operatorname{gcd}\left(r_{i}, r_{d}\right) \tau_{i}$ in $\Phi_{M}$. Proposition 3.5 shows that the latter element is zero in $\Phi_{M}$. Thus, $\sum_{i=1}^{d-1} \operatorname{gcd}\left(r_{i}, r_{d}\right) S_{i}$ reduces in the connected component $\left(Q_{K}\right)_{k}^{0}$. Our additional hypothesis implies that this connected component is unipotent. This follows from [Bosch et al. 1990, Theorem 9.5.4] if the greatest common divisor of the multiplicities of the components of $\mathcal{X}_{k}^{\prime}$ is 1 and from [Liu et al. 2004, Proposition 7.1] in general. It follows that the image of $\left(Q_{K}\right)_{k}^{0}$ under the canonical map $\eta: \mathcal{A}_{K} \times \mathcal{O}_{K} \mathcal{O}_{L} \rightarrow \mathcal{A}_{L}$ is trivial.

Consider now the element $\sum_{i=1}^{d-1} \operatorname{gcd}\left(r_{i}, r_{d}\right) S_{i}$ in $\operatorname{Jac}\left(X_{L}\right)(L)$. Our discussion above shows that it reduces to the element $r_{d}\left(\sum_{i=1}^{d-1} r_{i}\left(P_{i}-P_{d}\right)\right)$ in $\operatorname{Jac}\left(\mathcal{Y}_{k}\right)(k)$. We have thus proved that $r_{d}\left(\sum_{i=1}^{d-1} r_{i}\left(P_{i}-P_{d}\right)\right)=0$ in $\operatorname{Jac}\left(\mathcal{Y}_{k}\right)(k)$. Our hypothesis 
on the quotient of $\mathcal{Y}_{k}$ by $H$ implies that each $P_{i}-P_{d}$ has order $p$ (Proposition 2.5). Since $r_{d}\left(\sum_{i=1}^{d-1} r_{i}\left(P_{i}-P_{d}\right)\right)=0$ and we assume that $p$ does not divide $r_{d}$, we can conclude that $\sum_{i=1}^{d-1} r_{i}\left(P_{i}-P_{d}\right)=0$. Then Proposition 2.5 implies that $p$ divides $r_{i}$ for all $i=1, \ldots, d-1$. Since $\left|C_{0} \cdot C_{0}\right| p=r_{1}+\cdots+r_{d}$, it follows that $p$ divides $r_{d}$, which contradicts our assumption.

When the quotient of $\mathcal{Y}_{k}$ by the action of $H$ has positive genus, the image of $\left(Q_{K}\right)_{k}^{0}$ under the canonical map $\eta: \mathcal{A}_{K} \times \mathcal{O}_{K} \mathcal{O}_{L} \rightarrow \mathcal{A}_{L}$ is not trivial, and the following additional considerations must be discussed. Let $\operatorname{Norm}\left(\mathcal{X}^{\prime}\right)$ denote the normalization of $\mathcal{X}^{\prime}$ in the field of fractions of $\mathcal{Y}$. Since $\mathcal{Y}$ is integral over $\mathcal{Z}$, we have a natural map $\operatorname{Norm}\left(\mathcal{X}^{\prime}\right) \rightarrow \mathcal{Y}$. All components of $\mathcal{X}^{\prime}$ are rational except possibly the component $C_{0}$ [Lorenzini 2013a, Lemma 2.10].

By construction, we have a natural map $\operatorname{Norm}\left(\mathcal{X}^{\prime}\right) \rightarrow \mathcal{X}^{\prime} \times \mathcal{O}_{K} \mathcal{O}_{L}$. Let $\mathcal{N} \rightarrow$ $\operatorname{Norm}\left(\mathcal{X}^{\prime}\right)$ denote a resolution of the singularities of $\operatorname{Norm}\left(\mathcal{X}^{\prime}\right)$. Consider the commutative diagram of $\mathcal{O}_{L}$-morphisms

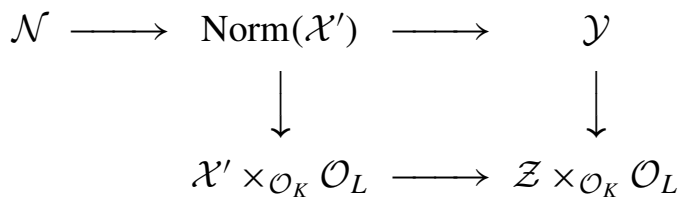

The maps $\mathcal{N} \rightarrow \operatorname{Norm}\left(\mathcal{X}^{\prime}\right) \rightarrow \mathcal{X}^{\prime} \times \mathcal{O}_{K} \mathcal{O}_{L}$ induce maps of the associated Picard functors

$$
\operatorname{Pic}_{\mathcal{X}^{\prime} / \mathcal{O}_{K}} \times \mathcal{O}_{K} \mathcal{O}_{L} \cong \operatorname{Pic}_{\mathcal{X}^{\prime} \times \mathcal{O}_{K}} \mathcal{O}_{L} / \mathcal{O}_{L} \rightarrow \operatorname{Pic}_{\operatorname{Norm}\left(\mathcal{X}^{\prime}\right) / \mathcal{O}_{L}} \rightarrow \operatorname{Pic}_{\mathcal{N} / \mathcal{O}_{L}}
$$

whose composition induces the canonical map of Néron models

$$
\eta: Q_{K} \times \mathcal{O}_{K} \mathcal{O}_{L} \rightarrow Q_{L}
$$

Considering the special fibers over $k$, we obtain a commutative diagram

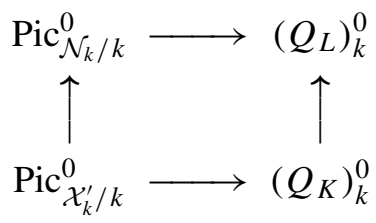

Since we do not have additional information on the special fiber $\mathcal{X}_{k}^{\prime}$, we cannot conclude that the bottom horizontal map is an isomorphism. It is however faithfully flat [Raynaud 1970, Corollaire 4.1.2]. Since the special fiber of $\mathcal{Y}$ is reduced, we find that the top horizontal map is an isomorphism [Bosch et al. 1990, Theorem 9.5.4].

Let $D$ denote the irreducible component of $\mathcal{N}_{k}$ lying above $\mathcal{Y}_{k}$. The composition $D \hookrightarrow \mathcal{N}_{k} \rightarrow \mathcal{Y}_{k}$ is an isomorphism. The image of $D$ in $\left(\mathcal{X}^{\prime}\right)_{k}^{\text {red }}$ is the curve $C_{0}$, and we will identify the map $D \rightarrow C_{0}$ with the quotient map $\rho: \mathcal{Y}_{k} \rightarrow \mathcal{Y}_{k} /\langle\sigma\rangle$. Consider the following diagram, whose top right horizontal morphism is an isomorphism: 


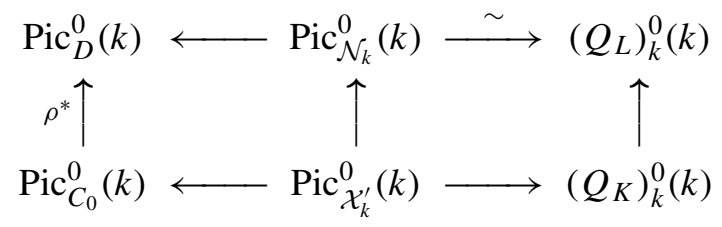

We may now conclude the proof of Theorem 5.3 using the same method as in the case where the reduction of $\operatorname{Jac}(X) / K$ is purely unipotent. Consider again the element $\sum_{i=1}^{d-1} \operatorname{gcd}\left(r_{i}, r_{d}\right) S_{i}$ in $\operatorname{Jac}(X)(K)$, which reduces to the element $\sum_{i=1}^{d-1} \operatorname{gcd}\left(r_{i}, r_{d}\right) \tau_{i}$ in $\Phi_{M}$. Proposition 3.5 shows that the latter element is zero in $\Phi_{M}$. Thus, $\sum_{i=1}^{d-1} \operatorname{gcd}\left(r_{i}, r_{d}\right) S_{i}$ reduces in the connected component $\left(Q_{K}\right)_{k}^{0}$. Consider now the element $\sum_{i=1}^{d-1} \operatorname{gcd}\left(r_{i}, r_{d}\right) S_{i}$ in $\operatorname{Jac}\left(X_{L}\right)(L)$. Our discussion above shows that it reduces to the element $r_{d}\left(\sum_{i=1}^{d-1} r_{i}\left(P_{i}-P_{d}\right)\right)$ in $\operatorname{Jac}\left(\mathcal{Y}_{k}\right)(k)$.

Since $\mathrm{Pic}_{\mathcal{X}_{k}^{\prime} / k}^{0} \rightarrow\left(Q_{K}\right)_{k}^{0}$ is a faithfully flat morphism and each of the above squares commutes, the element $\sum_{i=1}^{d-1} \operatorname{gcd}\left(r_{i}, r_{d}\right) S_{i}$, which reduces to $r_{d}\left(\sum_{i=1}^{d-1} r_{i}\left(P_{i}-P_{d}\right)\right)$ in $\operatorname{Pic}_{\mathcal{Y}_{k} / k}^{0}(k)$, in fact reduces to an element in $\rho^{*}\left(\operatorname{Jac}\left(\mathcal{Y}_{k} /\langle\sigma\rangle\right)\right)$. Thus, the image of $r_{d}\left(\sum_{i=1}^{d-1} r_{i}\left(P_{i}-P_{d}\right)\right)$ in $\operatorname{Jac}\left(\mathcal{Y}_{k}\right) / \rho^{*}\left(\operatorname{Jac}\left(\mathcal{Y}_{k} /\langle\sigma\rangle\right)\right)$ is trivial. Each $P_{i}-P_{d}$ defines an element of order $p$ in $\operatorname{Jac}\left(\mathcal{Y}_{k}\right) / \rho^{*}\left(\operatorname{Jac}\left(\mathcal{Y}_{k} /\langle\sigma\rangle\right)\right)$ (Proposition 2.5). Since $r_{d}\left(\sum_{i=1}^{d-1} r_{i}\left(P_{i}-P_{d}\right)\right)=0$, we conclude that $\sum_{i=1}^{d-1} r_{i}\left(P_{i}-P_{d}\right)=0$. Then Proposition 2.5 implies that $p$ divides $r_{i}$ for all $i=1, \ldots, d-1$, and since $\left|C_{0} \cdot C_{0}\right| p=r_{1}+\cdots+r_{d}$, we find that $p$ divides $r_{d}$, which contradicts our assumption.

Now that we know that $p$ divides $r_{i}$, we see that the multiplicities on the chain of $G$ that leaves $C_{0}$ starting with $D_{i}$ can only be increasing or constant because this chain of vertices of degree 2 contains no vertex of self-intersection -1 . If $D_{i}$ is not a node of $G$, we continue along this chain and find either a terminal vertex or a node of $G$. We cannot find a terminal vertex because the multiplicity of a terminal vertex can only be at most the multiplicity of its unique neighbor with equality only if the self-intersection of the terminal vertex is -1 . Thus, $G_{Q_{i}}$ contains a node of $G$.

Remark 5.4. Let $N_{i}$ denote the intersection matrix of the exceptional divisor, with smooth components and normal crossings, of a resolution of the $\mathbb{Z} / p \mathbb{Z}$-quotient singularity $Q_{i}$. We recall here some properties of $N_{i}$ :

(a) It is negative definite (attributed to Du Val in [Lipman 1969, Lemma 14.1]).

(b) The graph $G\left(N_{i}\right)$ associated with $N_{i}$ is a tree, and all components of the exceptional divisor are rational [Lorenzini 2013a, Theorem 2.8].

(c) Let $n_{i}$ denote the number of irreducible components in the exceptional divisor. The Smith group $\Phi_{N_{i}}:=\mathbb{Z}^{n_{i}} / \operatorname{Im}\left(N_{i}\right)$ is killed by $p$ [Lorenzini 2013a, Theorem 2.6].

(d) The fundamental cycle $Z$ of $N_{i}$ is such that $\left|Z^{2}\right| \leq p$ [Lorenzini 2013a, Theorem 2.3, Remark 2.4]. 


\section{The weakly ramified case}

We present in this section some applications of Theorem 5.3. Let us recall our notation. Let $K$ be a complete discrete valuation field with valuation $v$, ring of integers $\mathcal{O}_{K}$, uniformizer $\pi_{K}$ and residue field $k$ of characteristic $p>0$, assumed to be algebraically closed. Let $X / K$ be a smooth proper geometrically connected curve of genus $g>0$. When $g=1$, we assume in addition that $X(K) \neq \varnothing$.

Assume that $X / K$ does not have semistable reduction over $\mathcal{O}_{K}$ and that it achieves good reduction after a cyclic extension $L / K$ of prime degree $p$. Let $H=\langle\sigma\rangle$ denote the Galois group of $L / K$. Let $\mathcal{Y} / \mathcal{O}_{L}$ be the smooth model of $X_{L} / L$. Let $\mathcal{Z} / \mathcal{O}_{K}$ denote the quotient $\mathcal{Y} / H$ with singular points $Q_{1}, \ldots, Q_{d}$ and $d \geq 1$. Recall the regular model $f: \mathcal{X}^{\prime} \rightarrow \mathcal{Z}$ introduced in 5.2.

6.1. The resolution of a singularity $Q$ of $\mathcal{Z}$ is a local process and depends only on the local ring $\mathcal{O}_{\mathcal{Z}, Q}$. It seems therefore natural to try to relate the "complexity" of the resolution graph to some local invariants of $\mathcal{O}_{\mathcal{Z}, Q}$. In this respect, we propose the following.

Consider the Galois morphism $\rho: \mathcal{Y}_{k} \rightarrow \mathcal{Y}_{k} /\langle\sigma\rangle$. Associated with any point $Q \in \mathcal{Y}_{k} /\langle\sigma\rangle$ is the following measure of the ramification of $\rho$ over $Q$ :

$$
\nu(Q):=\delta(P)=\sum_{j=0}^{\infty}\left(\left|H_{j}(P)\right|-1\right),
$$

where $P$ is the preimage of $Q$ in $\mathcal{Y}_{k}$ and $H_{j}(P)$ denotes the $j$-th higher ramification group at $P$. (For more general morphisms, we would define $v(Q):=$ $\sum_{P \in \rho^{-1}(Q)} \delta(P)$.) Recall from 2.2 that the morphism is weakly ramified at $P$ if $\delta(P)=2(p-1)$. Our guess is that $v(Q)$ should also be an important measure of how complicated the exceptional divisor of the resolution of $Q$ is. To formulate this guess more precisely, we compare the expressions of the genus $g$ in the RiemannHurwitz formula and in the adjunction formula. The Riemann-Hurwitz formula for the morphism $\rho$ can be rephrased as

$$
2 g=2 g\left(\mathcal{Y}_{k}\right)=2|H| g\left(C_{0}\right)-2(|H|-1)+\sum_{i=1}^{d} v\left(Q_{i}\right) .
$$

Consider now the model $\mathcal{X}^{\prime}$. By hypothesis, it is minimal with the property that the special fiber has smooth components and normal crossings. Thus, none of the vertices $A$ in the graph $G:=G\left(\mathcal{X}^{\prime}\right)$ with degree 1 or 2 can have selfintersection -1 (we use here also the fact that only the curve $C_{0}$ can have positive genus [Lorenzini 2013a, Lemma 2.10]). Moreover, since the curve $X / K$ has potentially good reduction, the graph $G\left(\mathcal{X}^{\prime}\right)$ is a tree [Lorenzini 2013a, Lemma 2.10]. 
The adjunction formula

$$
2 g-2=\mathcal{X}_{k}^{\prime} \cdot \mathcal{X}_{k}^{\prime}+\mathcal{X}_{k}^{\prime} \cdot \Omega,
$$

with $\Omega$ a relative canonical divisor of $\mathcal{X}^{\prime} / \mathcal{O}_{K}$, can be rewritten as

$$
\begin{aligned}
2 g & =2|H| g\left(C_{0}\right)+\sum_{\text {vertex } A \text { of } G}(r(A)-1)\left(d_{G}(A)-2\right) \\
& =2|H| g\left(C_{0}\right)-2(|H|-1)+\sum_{i=1}^{d}\left(|H|-1+\sum_{\text {vertex } A \text { of } G_{Q_{i}}}(r(A)-1)\left(d_{G}(A)-2\right)\right) \\
& =2|H| g\left(C_{0}\right)-2(|H|-1)+\sum_{i=1}^{d} \gamma_{D_{i}} g_{D_{i}},
\end{aligned}
$$

where $D_{1}, \ldots, D_{d}$ are the vertices attached to $C_{0}$ in the tree $G\left(\mathcal{X}^{\prime}\right)$ and the integers $\gamma_{D_{i}}$ and $g_{D_{i}}$ are defined as in 4.1 and (4.1.2). Since the graph $G_{D_{i}}$ is nothing but the graph $G_{Q_{i}}$ of the desingularization of $Q_{i}$, we define our measure of the desingularization of $Q_{i}$ to be $\gamma_{Q_{i}} g_{Q_{i}}:=\gamma_{D_{i}} g_{D_{i}}$ for each $i=1, \ldots, d$. The integer $g_{Q_{i}}:=g_{D_{i}}$ depends only on the intersection matrix of the desingularization and the marked vertex $D_{i}$ on its graph. Since $r\left(C_{0}\right)=p$ and is divisible by $\gamma_{Q_{i}}$, we find that $\gamma_{Q_{i}}=1$ or $p$.

6.2. Our guess regarding the resolution $\mathcal{X}^{\prime} \rightarrow \mathcal{Z}$ of the singularities of $\mathcal{Z}$ is that

$$
\gamma_{Q_{i}} g_{Q_{i}}=v\left(Q_{i}\right) \text { holds for all } i=1, \ldots, d .
$$

This equality would have interesting implications. For instance, since $H=\mathbb{Z} / p \mathbb{Z}$, we always have $v(Q)$ divisible by $p-1$ so that $p-1$ divides $\gamma_{Q_{i}} g_{Q_{i}}$ when $\gamma_{Q_{i}} g_{Q_{i}}=v\left(Q_{i}\right)$. Since $\gamma_{Q_{i}}=1$ or $p$, we find that

$$
p-1 \text { divides } g_{Q_{i}} \text { when } \gamma_{Q_{i}} g_{Q_{i}}=v\left(Q_{i}\right) \text {. }
$$

Examples where $g_{Q_{i}}=2(p-1)$ and $3(p-1)$ are given in 4.7 and Remark 4.9. It immediately follows from the Riemann-Hurwitz formula and the adjunction formula that:

Lemma 6.3. With the above notation and hypotheses,

$$
\sum_{i=1}^{d} v\left(Q_{i}\right)=\sum_{i=1}^{d} \gamma_{Q_{i}} g_{Q_{i}} .
$$

We now prove the equality $\gamma_{Q_{i}} g_{Q_{i}}=v\left(Q_{i}\right)=2(p-1)$ for all $i=1, \ldots, d$ in the weakly ramified case, using Theorem 5.3. 
Theorem 6.4. Let $X / K$ be a curve with potentially good reduction after a ramified extension $L / K$ of prime degree $p$. Keep the above notation. Then for all $i=1, \ldots, d$ :

(a) We have $\gamma_{Q_{i}} g_{Q_{i}} \geq 2(p-1)$ and $v\left(Q_{i}\right) \geq 2(p-1)$.

(b) If the ramification points of $\mathcal{Y}_{k} \rightarrow \mathcal{Y}_{k} /\langle\sigma\rangle$ are all weakly ramified (in particular, if $\mathcal{Y}_{k}$ is ordinary), then $\gamma_{Q_{i}} g_{Q_{i}}=v\left(Q_{i}\right)=2(p-1)$.

Proof. (a) The fact that $v\left(Q_{i}\right) \geq 2(p-1)$ follows immediately from the properties of a wildly ramified extension: the higher ramification groups $H_{0}$ and $H_{1}$ must be nontrivial. To prove that $\gamma_{Q_{i}} g_{Q_{i}} \geq 2(p-1)$, we note first that Theorem 5.3 shows that $p \mid r_{i}$. The inequality follows then from Proposition 4.3.

(b) When the ramification points of $\mathcal{Y}_{k} \rightarrow \mathcal{Y}_{k} /\langle\sigma\rangle$ are all weakly ramified, we have $v\left(Q_{i}\right)=2(p-1)$ (2.2). It follows from (6.3.1) and from the fact that $\gamma_{Q_{i}} g_{Q_{i}} \geq$ $2(p-1)$ proven in (a) that $\gamma_{Q_{i}} g_{Q_{i}}=2(p-1)$.

Remark 6.5. Without the use of Theorem 5.3, we could only argue that $\gamma_{Q_{i}} g_{Q_{i}} \geq$ $p-1$. Indeed, if $r\left(C_{0}\right)$ does not divide $r\left(D_{i}\right)$, then $\gamma_{D_{i}}=1$. Then we can use the fact that $g_{Q_{i}} \geq r\left(C_{0}\right)-1$ established in Remark 4.10.

Using the notation $\gamma_{Q_{i}}$ introduced in this section, we may now state a corollary to Theorem 5.3.

Corollary 6.6. Let $X / K$ be a curve with potentially good reduction after a wildly ramified Galois extension $L / K$ of degree $p$ as in Theorem 5.3. Let $N_{i}$ denote the intersection matrix associated with the resolution of $Q_{i}$. Assume that $\gamma_{Q_{i}}=1$. Then $p^{2}$ divides $\operatorname{det}\left(N_{i}\right)$.

Proof. The graph associated with the matrix $N_{i}$ is $G_{Q_{i}}$ with a marked vertex $D_{i}$ on it. Let $R_{D_{i}}$ denote the vector of multiplicities of the components of the resolution of $Q_{i}$. Then the determinant of $N_{i}$ can be computed in terms of the coefficients of $R_{D_{i}} / \gamma_{D_{i}}$ (see [Lorenzini 2013a, Theorem 3.14]). In particular, it is known that $\left(r\left(C_{0}\right) / \gamma_{D_{i}}\right) \operatorname{gcd}\left(r\left(C_{0}\right) / \gamma_{D_{i}}, r\left(D_{i}\right) / \gamma_{D_{i}}\right)$ divides $\operatorname{det}\left(N_{i}\right)$. Under our hypotheses, $r\left(C_{0}\right)=p, p$ divides $r\left(D_{i}\right)$ (Theorem 5.3) and $\gamma_{D_{i}}=1$.

Remark 6.7. Let $X / K$ be a curve with potentially good reduction after a wildly ramified extension $L / K$ of degree $p$ as in Theorem 5.3. Let $N_{i}$ denote the intersection matrix associated with the resolution of $Q_{i}$. Then $p$ kills the Smith group $\Phi_{N_{i}}$ [Lorenzini 2013a, Theorem 2.6], and thus, $\left|\operatorname{det}\left(N_{i}\right)\right|$ is a power of $p$. It follows from (4.10.3) that $\operatorname{ord}_{p}\left(\left|\operatorname{det}\left(N_{i}\right)\right|\right)(p-1) \leq g_{D_{i}}$.

In the examples of graphs and matrices $N_{i}$ given in Remark 4.9 with $g_{D_{i}}=$ $3(p-1)$, we find that both $\left|\operatorname{det}\left(N_{i}\right)\right|=p^{2}$ and $\left|\operatorname{det}\left(N_{i}\right)\right|=p^{3}$ can occur: the former in (b) and (c)(ii), and the latter in (a) and (c)(i). 
Theorem 6.8. Let $X / K$ be a curve with potentially good reduction after a wildly ramified Galois extension $L / K$ of degree $p$. Assume that all ramification points of $\mathcal{Y}_{k} \rightarrow \mathcal{Y}_{k} /\langle\sigma\rangle$ are weakly ramified (this is the case if $\mathcal{Y}_{k}$ is ordinary). Keep the above notation. Then, for all $i=1, \ldots, d$, we have $r_{i}=p$, and $G_{Q_{i}}$ is a graph with a single node $C_{i}$ of degree 3 :

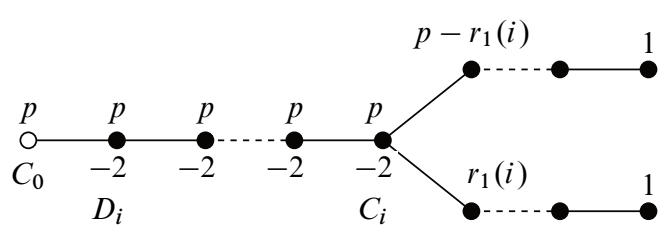

The intersection matrix $N\left(p, \alpha_{i}, r_{1}(i)\right)$ of the resolution of $Q_{i}$ is uniquely determined as in 4.7 by the two integers $\alpha_{i}$ and $r_{1}(i)$ with $1 \leq r_{1}(i)<p$. The integer $\alpha_{i}$ is the number of vertices of self-intersection -2 (including the node $C_{i}$ ) on the chain in $G_{Q_{i}}$ connecting the node $C_{0}$ to the single node $C_{i}$ of $G_{Q_{i}}$, and this integer $\alpha_{i}$ is divisible by $p$.

Proof. Theorem 6.4(b) shows that $\gamma_{Q_{i}} g_{Q_{i}}=2(p-1)$ for all $i=1, \ldots, d$. Proposition 4.3 classifies the graphs with $\gamma_{Q_{i}} g_{Q_{i}}=2(p-1)$, and the statement on the shape of the graph follows.

The Smith group of the intersection matrix $N\left(p, \alpha_{i}, r_{1}(i)\right)$ is computed in [Lorenzini 2013a, \$3.19, Lemma 3.21] and is found to be of order $p^{2}$ and killed by $p$ if and only if $p$ divides $\alpha_{i}$. Theorem 2.6(c) of [Lorenzini 2013a] shows that this Smith group must be killed by $p$. The divisibility $p \mid \alpha_{i}$ follows.

Remark 6.9. It is natural to wonder whether the statements of Theorems 6.4(b) and 6.8 hold for the resolution of $Q_{i}$ when $P_{i}$ is a weakly ramified ramification point of $\mathcal{Y}_{k} \rightarrow \mathcal{Y}_{k}\langle\sigma\rangle$ without also assuming as we do in Theorems 6.4(b) and 6.8 that all ramification points are weakly ramified.

Corollary 6.10. Let $X / K$ be a curve with potentially good reduction after a wildly ramified Galois extension $L / K$ of degree $p$ as in Theorem 6.8. Suppose that $g>1$ and that all ramification points of $\mathcal{Y}_{k} \rightarrow \mathcal{Y}_{k} /\langle\sigma\rangle$ are weakly ramified. Then:

(a) $X(K) \neq \varnothing$.

(b) Let $A / K$ denote the Jacobian of $X / K$. Let $\mathcal{A} / \mathcal{O}_{K}$ be its Néron model. Then the unipotent part $U / k$ of the connected component of the identity in $\mathcal{A}_{k} / k$ is a product of additive groups $\mathbb{G}_{a, k}$.

(c) The group of components $\Phi_{A, K}$ of the Néron model is isomorphic to $(\mathbb{Z} / p \mathbb{Z})^{2 d-2}$.

${ }^{1}$ A bullet $\bullet$ represents an irreducible component of the desingularization of $Q_{i}$. A positive number next to a vertex is the multiplicity of the corresponding component while a negative number next to a vertex is the self-intersection of the component. 
Proof. Part (a) is immediate since it follows from Theorem 6.8 that a regular model of $X / K$ contains a component of multiplicity 1 . It follows from [Penniston 2000, Theorem 2.4] that $p$ kills $U$ since the maximal multiplicity in the regular model $\mathcal{X}^{\prime} / \mathcal{O}_{K}$ is equal to $p$. That $U$ is now split follows from [Serre 1959, Proposition VII.11.11]. This proves (b).

The order of $\Phi_{A, K}$ can be computed using the intersection matrix of the regular model $\mathcal{X}^{\prime}$. Since the associated graph is a tree, we find using [Lorenzini 1989, Corollary 2.5] that $\left|\Phi_{A, K}\right|=p^{2 d-2}$. Part (c) follows since $\Phi_{A, K}$ is killed by $[L: K]$ because $A / K$ has potentially good reduction [Edixhoven et al. 1996].

Note that in general the special fiber $\mathcal{A}_{k} / k$ need not be killed by $p$ even when its subgroup $U$ and quotient $\Phi_{A, K}$ are both killed by $p$ (see [Liu and Lorenzini 2001] for a general discussion of such phenomena).

6.11. Let $A / K$ be the Jacobian of a smooth proper and geometrically connected curve $X / K$ having a $K$-rational point. For use in our next corollary, we recall below the main result of [Bosch and Lorenzini 2002, Theorem 4.6]. Identify $A / K$ with its dual $A^{\prime} / K$ via the map $-\varphi_{[\Theta]}: A \rightarrow A^{\prime}$ as in [Bosch and Lorenzini 2002] just before Theorem 4.6. Let $\mathcal{X} / \mathcal{O}_{K}$ denote a regular model of $X / K$. Let $M$ be the intersection matrix of $\mathcal{X}_{k}$. Identify, as recalled in [Bosch and Lorenzini 2002, Theorem 2.3], the component group $\Phi_{A / K}$ with the group of components $\Phi_{M}$ of $M$ $\left(\Phi_{M}\right.$ is the torsion subgroup of $\mathbb{Z}^{v} / \operatorname{Im}(M)$ ). Then Grothendieck's pairing

$$
\langle\cdot, \cdot\rangle_{K}: \Phi_{A / K} \times \Phi_{A / K} \rightarrow \mathbb{Q} / \mathbb{Z}
$$

coincides with the pairing $\langle\cdot, \cdot\rangle_{M}: \Phi_{A / K} \times \Phi_{A / K} \rightarrow \mathbb{Q} / \mathbb{Z}$ considered in 3.1. In particular, this pairing is nondegenerate. Recall also the definition of the functorial subgroup $\Phi_{A / K}^{0}$ of $\Phi_{A / K}$ in 1.3. We denote by $\left(\Phi_{A / K}^{0}\right)^{\perp}$ the orthogonal of $\Phi_{A / K}^{0}$ under Grothendieck's pairing.

Corollary 6.12. Let $A / K$ be the Jacobian of a curve $X / K$ of genus $g>1$ having potentially good reduction after a Galois extension $L / K$ of degree $p$ as in Theorem 6.8. Assume that all ramification points of $\mathcal{Y}_{k} \rightarrow \mathcal{Y}_{k} /\langle\sigma\rangle$ are weakly ramified. Then $\Phi_{A / K}$ is a $\mathbb{Z} / p \mathbb{Z}$-vector space of dimension $2 d-2$, and $\Phi_{A / K}^{0}$ is a subspace of dimension $d-1$. Moreover, $\Phi_{A / K}^{0}=\left(\Phi_{A / K}^{0}\right)^{\perp}$.

Proof. It follows from Corollary 6.10 that $X(K) \neq \varnothing$. We can thus use the results of [Bosch and Lorenzini 2002] recalled above. We produce below explicit generators for the groups $\Phi_{A / K}$ and $\Phi_{A / K}^{0}$. For each singular point $Q_{i}$ on the model $\mathcal{Z} / \mathcal{O}_{K}$, denote by $A_{i}$ and $B_{i}$ the terminal components of multiplicity 1 in the exceptional divisor of the resolution of $Q_{i}$ in $\mathcal{X}^{\prime}$. Let $\boldsymbol{\alpha}_{i}$ denote the image in $\Phi_{A / K}$ of the vector $E\left(A_{i}, B_{i}\right), i=1, \ldots, d-1$ (notation as in 3.1). Let $\boldsymbol{\beta}_{i}$ denote the image in $\Phi_{A / K}$ of the vector $E\left(A_{i}, A_{d}\right), i=1, \ldots, d-1$. We have seen in Corollary 6.10 that $\Phi_{A / K}$ is a $\mathbb{Z} / p \mathbb{Z}$-vector space of dimension $2(d-1)$. 
We claim that

$$
\left\{\boldsymbol{\alpha}_{1}, \ldots, \boldsymbol{\alpha}_{d-1}, \boldsymbol{\beta}_{1}, \ldots, \boldsymbol{\beta}_{d-1}\right\}
$$

is a basis for $\Phi_{A / K}$ and that $\left\{\boldsymbol{\alpha}_{1}, \ldots, \boldsymbol{\alpha}_{d-1}\right\}$ is a basis for $\Phi_{A / K}^{0}$. To prove our claim, consider the matrix $V:=\left(\left\langle\boldsymbol{\alpha}_{i}, \boldsymbol{\beta}_{j}\right\rangle\right)_{1 \leq i, j \leq d-1}$ with coefficients in $\mathbb{Q} / \mathbb{Z}$. We can use the computation (4.8.1) to show that $V$ is the diagonal matrix

$$
\operatorname{diag}\left(c_{1} / p(\bmod \mathbb{Z}), \ldots, c_{d-1} / p(\bmod \mathbb{Z})\right),
$$

where, for each $i=1, \ldots, d-1,0<c_{i}<p$ and $p$ divides $c_{i} r_{1}(i)-1$. In particular, $c_{i} / p \neq 0$ in $\mathbb{Q} / \mathbb{Z}$. It follows that the set $\left\{\boldsymbol{\alpha}_{1}, \ldots, \boldsymbol{\alpha}_{d-1}, \boldsymbol{\beta}_{1}, \ldots, \boldsymbol{\beta}_{d-1}\right\}$ is linearly independent in $(\mathbb{Z} / p \mathbb{Z})^{2 d-2}$. Hence, it is a basis.

It follows from the explicit computations in [Lorenzini 2000, Proposition 3.7(a)], that $\left\langle\boldsymbol{\alpha}_{i}, \boldsymbol{\alpha}_{j}\right\rangle=0$ for all $1 \leq i, j \leq d-1$. Since the pairing $\langle\cdot, \cdot\rangle$ is perfect on $(\mathbb{Z} / p \mathbb{Z})^{2 d-2}$, we find that $\left\{\boldsymbol{\alpha}_{1}, \ldots, \boldsymbol{\alpha}_{d-1}\right\}$ generates a maximal isotropic subspace.

It remains to show that $\boldsymbol{\alpha}_{1}, \ldots, \boldsymbol{\alpha}_{d-1}$ belong to $\Phi_{A / K}^{0}$ and that neither $\boldsymbol{\beta}_{1}, \ldots$, $\boldsymbol{\beta}_{d-1}$ nor any nontrivial linear combination of $\boldsymbol{\beta}_{1}, \ldots, \boldsymbol{\beta}_{d-1}$ belong to $\Phi_{A / K}^{0}$. For this, since $K$ is complete, we can pick for each $i=1, \ldots, d-1$ two $K$-rational points $a_{i}$ and $b_{i}$ of $X$ whose closure in $\mathcal{X}^{\prime}$ intersects $\mathcal{X}_{k}^{\prime}$ in a smooth point of $A_{i}$ and $B_{i}$, respectively (see, e.g., [Bosch et al. 1990, Corollary 9.1.9]). Then $a_{i}-b_{i}$ and $a_{i}-a_{d}$ are divisors of degree 0 on $X$, which we identify with $K$-rational points in the Jacobian $A / K$ of $X / K$. These rational points reduce in the component group $\Phi_{A / K}$ of the Néron model of $A / K$ to the points $\boldsymbol{\alpha}_{i}$ and $\boldsymbol{\beta}_{i}$, respectively. Since $A(K) \subset A(L)$, we can reduce $a_{i}-b_{i}$ in the special fiber of the Néron model $\mathcal{A}^{\prime} / \mathcal{O}_{L}$. This special fiber is isomorphic to the Jacobian of the special fiber $\mathcal{Y}_{k}$ of the smooth model $\mathcal{Y} / \mathcal{O}_{L}$ of $X_{L} / L$. It is clear that, by construction, the reduction of $a_{i}-b_{i}$ is trivial so that $\alpha_{i} \in \Phi_{A / K}^{0}$ for $i=1, \ldots, d-1$. On the other hand, the reduction of $a_{i}-a_{d}$ is the divisor $P_{i}-P_{d}$, which is a nontrivial $p$-torsion point when viewed in the quotient $\mathcal{A}_{k}^{\prime} / \eta\left(\mathcal{A}_{k}\right)$. This shows that $\boldsymbol{\beta}_{i} \notin \Phi_{A / K}^{0}$ for $i=1, \ldots, d-1$. Moreover, any nontrivial linear combination of the images of the divisors $P_{i}-P_{d}$ is not zero in $\mathcal{A}_{k}^{\prime} / \eta\left(\mathcal{A}_{k}\right)$ (Proposition 2.5), so no nontrivial linear combination of $\boldsymbol{\beta}_{1}, \ldots, \boldsymbol{\beta}_{d-1}$ belongs to $\Phi_{A / K}^{0}$.

Example 6.13. Examples of curves having good reduction after an extension of degree $p$ can be obtained as twists as follows. Choose a smooth proper curve $C / k$ having an automorphism $\sigma_{k}$ of order $p$. Over an appropriate ring $\mathcal{O}_{K}$ with residue field $k$, there exists a smooth scheme $\mathcal{Y}^{0} / \mathcal{O}_{K}$ with an $\mathcal{O}_{K}$-automorphism $\sigma$ such that $C$ is $k$-isomorphic to $\mathcal{Y}_{k}^{0}$ and $\sigma$ restricted to $\mathcal{Y}_{k}^{0}$ induces the given automorphism $\sigma_{k}$. It is shown in [Sekiguchi et al. 1989, §IV, Theorem 2.2] that one can take $\mathcal{O}_{K}$ to be the Witt ring $W(k)\left(\zeta_{p}\right)$ with $\zeta_{p}$ a primitive $p$-th root of unity. If one wants a lift in equicharacteristic $p$, one can trivially take $\mathcal{O}_{K}=k \llbracket t \rrbracket$. 
Choose any cyclic (ramified) extension $L / K$ of degree $p$. The twist of $\mathcal{Y}_{K}^{0} / K$ by $L / K$ and $\sigma$ is a curve $X / K$ that achieves good reduction over $L$. Starting with an ordinary curve $C / k$ produces a curve $X / K$ having potentially good ordinary reduction over $L$.

Corollary 6.14. Fix any odd prime $p$. For each integer $m>0$, there exist a regular local ring $B$ of equicharacteristic $p$ endowed with an action of $H:=\mathbb{Z} / p \mathbb{Z}$ and $a$ regular local ring $B^{\prime}$ of mixed characteristic $(0, p)$ endowed with an action of $\mathbb{Z} / p \mathbb{Z}$ such that $\operatorname{Spec} B^{H}$ and $\operatorname{Spec}\left(B^{\prime}\right)^{H}$ are singular exactly at their closed point, and the graphs associated with a minimal resolution of $\operatorname{Spec} B^{H}$ and $\operatorname{Spec}\left(B^{\prime}\right)^{H}$ have one node and more than $m$ vertices.

Proof. As we noted in Example 6.13, there exist a field $K$ of either mixed characteristic $(0, p)$ or of equicharacteristic $p$ and a curve $X / K$ without good reduction over $K$ and with good ordinary reduction over a Galois extension $L / K$ of degree $p$. Let $H:=\operatorname{Gal}(L / K)$. Let $\mathcal{Y} / \mathcal{O}_{L}$ denote the smooth model of $X_{L} / L$. Let $\mathcal{Z} / \mathcal{O}_{K}$ denote the quotient $\mathcal{Y} / H$. Let $P$ denote a ramification point of the morphism $\mathcal{Y}_{k} \rightarrow \mathcal{Y}_{k} / H$, and let $B:=\mathcal{O}_{\mathcal{Y}, P}$. Theorem 6.8 shows that the resolution of singularity of $\operatorname{Spec} B^{H}$ has an intersection matrix of type $N\left(p, \alpha, r_{1}\right)$ for some $\alpha \geq 1$ and $0<r_{1}<p$.

Immediately after the statement of Theorem 6.8 given in the introduction, we briefly alluded to the fact that the integer $\alpha$ is likely to be related to the valuation of the different of $L / K$. Thus, in principle, by choosing $K$ and $L / K$ appropriately, the above method will produce examples with $\alpha$ as large, as desired. Since at this time we do not know how to prove in general that $\alpha$ is related to the valuation of the different of $L / K$ (except when $p=2$ and $g=1$; see [Lorenzini 2013a, Theorem 4.1]), we proceed below with a different argument to prove the existence of resolutions with $\alpha$ as large, as desired.

Consider a quadratic extension $K^{\prime} / K$. Since $p$ is odd by hypothesis, the extension $K^{\prime} / K$ is tame, and one knows how to compute a regular model of $X_{K^{\prime}} / K^{\prime}$ from the model $\mathcal{X} / \mathcal{O}_{K}$ of $X / K$ obtained in Theorem 6.8: simply normalize the base change $\mathcal{X} \times \mathcal{O}_{K} \mathcal{O}_{K^{\prime}}$ and resolve its singularities. A singularity on the normalization can only be the preimage of a closed point of $\mathcal{X}_{k}$ that belongs to two irreducible components of $\mathcal{X}_{k}$ and such that both components have odd multiplicity. This singular point is resolved by a single smooth rational curve.

Let $L^{\prime}:=L K^{\prime}$ with $\left[L^{\prime}: K^{\prime}\right]=p$. The curve $X_{K^{\prime}} / K^{\prime}$ achieves good ordinary reduction over $L^{\prime}$. The model $\mathcal{Y}^{\prime} / \mathcal{O}_{L^{\prime}}:=\mathcal{Y} \times \mathcal{O}_{L} \mathcal{O}_{L^{\prime}}$ is smooth, and we let $P^{\prime}$ denote the preimage of $P$ under the natural map $\mathcal{Y}^{\prime} \rightarrow \mathcal{Y}$. Let $B^{\prime}:=\mathcal{O}_{\mathcal{Y}^{\prime}, P^{\prime}}$. We leave it to the reader to check, using [Halle 2010, Proposition 4.3] and the desingularization of the normalization of $\mathcal{X} \times \mathcal{O}_{K} \mathcal{O}_{K^{\prime}}$, that the resolution of the singularity of $\operatorname{Spec}\left(B^{\prime}\right)^{H}$ has an intersection matrix of type $N\left(p, 2 \alpha, r_{1}^{\prime}\right)$, where $r_{1}^{\prime}:=r_{1} / 2$ if $r_{1}$ is even and $r_{1}^{\prime}:=\left(r_{1}+p\right) / 2$ if $r_{1}$ is odd. 
Since we can make an infinite chain of quadratic extensions $K \subset K^{\prime} \subset K^{\prime \prime} \subset \cdots$ and since the graph associated with $N\left(p, \beta, r_{1}\right)$ has at least $\beta$ irreducible components, the corollary is proved.

Remark 6.15. Consider an intersection matrix $N$, and assume that, for some prime $p$, it satisfies all the conditions listed in Remark 5.4, conditions that would have to be satisfied if this intersection matrix was associated with the resolution of a $\mathbb{Z} / p \mathbb{Z}$-singularity: its graph $G(N)$ is a tree, $|\operatorname{det}(N)|$ is a power of $p$, the Smith group $\Phi_{N}$ is killed by $p$ and the fundamental cycle $Z$ has $\left|Z^{2}\right| \leq p$. If $\operatorname{det}(N)=1$ and $G(N)$ is a tree, then the above conditions are satisfied for every prime at least equal to $\left|Z^{2}\right|$. In particular, when $\operatorname{det}(N)=1$, the matrix $N$ could potentially be associated with the resolution of a $\mathbb{Z} / p \mathbb{Z}$-singularity for infinitely many primes $p$.

An interesting consequence of our guess in 6.2 that $\gamma_{Q_{i}} g_{Q_{i}}=v\left(Q_{i}\right)$ holds for all $i=1, \ldots, d$ is that a matrix $N$ as above can be associated with the resolution of a $(\mathbb{Z} / p \mathbb{Z})$-quotient singularity $\mathcal{X}^{\prime} \rightarrow \mathcal{Z}$ occurring in models of curves as at the beginning of this section only for finitely many primes $p$. Indeed, the choice of a vertex $D$ on $N$ lets us define the integer $g_{D}$ associated with $N$ and $D$. If $N$ is the intersection matrix of the resolution of a singularity $Q_{i}$ of $\mathcal{Z}$ with the marked vertex $D$ linked to $C_{0}$, we noted in 6.2 that $p-1$ must then divide $g_{D}$ when the equality $\gamma_{Q_{i}} g_{Q_{i}}=v\left(Q_{i}\right)$ holds. Since there are only finitely many vertices $D$, the set of integers $g_{D}$ is finite, and hence, any prime $p$ larger than the maximum of the integers $g_{D}$ cannot have the property that $p-1$ divides some $g_{D}$.

Remark 6.16. Let $X / K$ be a curve with potentially good reduction over an extension $L / K$ of degree $p$ as at the beginning of this section. Let $Q_{i}$ be a singular point of the quotient $\mathcal{Z}$, and consider the graph $G_{Q_{i}}$ associated with the resolution of $Q_{i}$ in $\mathcal{X}^{\prime} \rightarrow \mathcal{Z}$. One may wonder whether a node of $G$ in $G_{Q_{i}}$ could have its multiplicity in $\mathcal{X}_{k}^{\prime}$ divisible by $p^{2}$. Similar considerations are found in [Lorenzini 2010, Question 1.4].

\section{Acknowledgements}

It is my pleasure to thank Qing Liu, Werner Lütkebohmert and Michel Raynaud for helpful comments. I also thank the referee for a careful reading of the article.

\section{References}

[Altman and Kleiman 1970] A. Altman and S. Kleiman, Introduction to Grothendieck duality theory, Lecture Notes in Mathematics 146, Springer, Berlin, 1970. MR 43 \#224 Zbl 0215.37201

[Artin 1975] M. Artin, "Wildly ramified Z/2 actions in dimension two", Proc. Amer. Math. Soc. 52 (1975), 60-64. MR 51 \#10336 Zbl 0315.14015

[Bosch and Lorenzini 2002] S. Bosch and D. Lorenzini, "Grothendieck's pairing on component groups of Jacobians”, Invent. Math. 148:2 (2002), 353-396. MR 2003b:14053 Zbl 1061.14042 
[Bosch et al. 1990] S. Bosch, W. Lütkebohmert, and M. Raynaud, Néron models, Ergebnisse der Mathematik und ihrer Grenzgebiete (3) 21, Springer, Berlin, 1990. MR 91i:14034 Zbl 0705.14001

[Edixhoven et al. 1996] B. Edixhoven, Q. Liu, and D. Lorenzini, "The $p$-part of the group of components of a Néron model", J. Algebraic Geom. 5:4 (1996), 801-813. MR 98m:14051 Zbl 0898.14007

[Gabber et al. 2013] O. Gabber, Q. Liu, and D. Lorenzini, "The index of an algebraic variety", Invent. Math. 192:3 (2013), 567-626. MR 3049930 Zbl 1268.13009

[Halle 2010] L. H. Halle, "Stable reduction of curves and tame ramification", Math. Z. 265:3 (2010), 529-550. MR 2011e:14030 Zbl 1195.14011

[Hasse 1934] H. Hasse, "Theorie der relativ-zyklischen algebraischen Funktionenkörper, insbesondere bei endlichem Konstantenkörper”, J. Reine Angew. Math. 172 (1934), 37-54. Zbl 0010.00501

[Katsura 1978] T. Katsura, "On Kummer surfaces in characteristic 2", pp. 525-542 in Proceedings of the International Symposium on Algebraic Geometry (Kyoto, 1977), edited by M. Nagata, Kinokuniya Book Store, Tokyo, 1978. MR 82d:14022 Zbl 0411.14010

[Lipman 1969] J. Lipman, "Rational singularities, with applications to algebraic surfaces and unique factorization", Inst. Hautes Études Sci. Publ. Math. 36 (1969), 195-279. MR 43 \#1986 Zbl 0181.48903

[Liu and Lorenzini 2001] Q. Liu and D. Lorenzini, "Special fibers of Néron models and wild ramification”, J. Reine Angew. Math. 532 (2001), 179-222. MR 2001m:11094 Zbl 0970.14025

[Liu et al. 2004] Q. Liu, D. Lorenzini, and M. Raynaud, "Néron models, Lie algebras, and reduction of curves of genus one", Invent. Math. 157:3 (2004), 455-518. MR 2005m:14039 Zbl 1060.14037

[Lorenzini 1989] D. Lorenzini, “Arithmetical graphs", Math. Ann. 285:3 (1989), 481-501. MR 91b: 14026 Zbl 0662.14008

[Lorenzini 1990a] D. Lorenzini, "Dual graphs of degenerating curves", Math. Ann. 287:1 (1990), 135-150. MR 91j:14018 Zbl 0668.14019

[Lorenzini 1990b] D. Lorenzini, "Groups of components of Néron models of Jacobians", Compositio Math. 73:2 (1990), 145-160. MR 92d:14019 Zbl 0737.14008

[Lorenzini 1993] D. Lorenzini, "On the group of components of a Néron model”, J. Reine Angew. Math. 445 (1993), 109-160. MR 94k:11065 Zbl 0781.14029

[Lorenzini 2000] D. Lorenzini, "Reduction of points in the group of components of the Néron model of a Jacobian”, J. Reine Angew. Math. 527 (2000), 117-150. MR 2001i:11073 Zbl 0983.14022

[Lorenzini 2010] D. Lorenzini, "Models of curves and wild ramification", Pure Appl. Math. Q. 6:1 (2010), 41-82. MR 2012a:11068 Zbl 1200.14052

[Lorenzini 2013a] D. Lorenzini, "Wild quotient singularities of surfaces", Math. Z. 275:1-2 (2013), 211-232. MR 3101805 Zbl 06224338

[Lorenzini 2013b] D. Lorenzini, "Wild quotients of products of curves", preprint, 2013, available at http://www.math.uga.edu/ lorenz/Paper3.pdf.

[Lorenzini and Tucker 2002] D. Lorenzini and T. J. Tucker, "Thue equations and the method of Chabauty-Coleman", Invent. Math. 148:1 (2002), 47-77. MR 2003d:11088 Zbl 1048.11023

[Penniston 2000] D. Penniston, "Unipotent groups and curves of genus two", Math. Ann. 317:1 (2000), 57-78. MR 2001b:14050 Zbl 1005.14010

[Peskin 1983] B. R. Peskin, "Quotient-singularities and wild p-cyclic actions", J. Algebra 81:1 (1983), 72-99. MR 84k:13018 Zbl 0517.14017

[Raynaud 1970] M. Raynaud, "Spécialisation du foncteur de Picard”, Inst. Hautes Études Sci. Publ. Math. 38 (1970), 27-76. MR 44 \#227 Zbl 0207.51602 
[Sekiguchi et al. 1989] T. Sekiguchi, F. Oort, and N. Suwa, "On the deformation of Artin-Schreier to Kummer”, Ann. Sci. École Norm. Sup. (4) 22:3 (1989), 345-375. MR 91g:14041 Zbl 0714.14024

[Serre 1959] J.-P. Serre, Groupes algébriques et corps de classes, Publications de l'institut de mathématique de l'université de Nancago, VII. Hermann, Paris, 1959. MR 21 \#1973 Zbl 0097.35604

[Singh 1974] B. Singh, "On the group of automorphisms of function field of genus at least two", $J$. Pure Appl. Algebra 4 (1974), 205-229. MR 50 \#13047 Zbl 0284.12007

[Subrao 1975] D. Subrao, "The p-rank of Artin-Schreier curves", Manuscripta Math. 16:2 (1975), 169-193. MR 51 \#12868 Zbl 0321.14017

[Viehweg 1977] E. Viehweg, "Invarianten der degenerierten Fasern in lokalen Familien von Kurven", J. Reine Angew. Math. 293/294 (1977), 284-308. MR 58 \#16655 Zbl 0349.14017

[Winters 1974] G. B. Winters, "On the existence of certain families of curves", Amer. J. Math. 96 (1974), 215-228. MR 50 \#9874 Zbl 0334.14004

Communicated by Brian Conrad

Received 2013-01-03 Revised 2013-06-06 Accepted 2013-07-16

lorenzin@uga.edu

Department of Mathematics, University of Georgia, Athens, GA 30602, United States 


\section{Algebra \& Number Theory}

msp.org/ant

\section{EDITORS}

MANAGING EDITOR

Bjorn Poonen

Massachusetts Institute of Technology

Cambridge, USA

\author{
EDITORIAL BOARD CHAIR \\ David Eisenbud \\ University of California \\ Berkeley, USA
}

\section{BOARD OF EDITORS}

Georgia Benkart

Dave Benson

Richard E. Borcherds

John H. Coates

J-L. Colliot-Thélène

Brian D. Conrad

Hélène Esnault

Hubert Flenner

Edward Frenkel

Andrew Granville

Joseph Gubeladze

Roger Heath-Brown

Ehud Hrushovski

Craig Huneke

Mikhail Kapranov

Yujiro Kawamata

János Kollár

Yuri Manin

Barry Mazur

Philippe Michel
University of Wisconsin, Madison, USA

University of Aberdeen, Scotland

University of California, Berkeley, USA

University of Cambridge, UK

CNRS, Université Paris-Sud, France

University of Michigan, USA

Freie Universität Berlin, Germany

Ruhr-Universität, Germany

University of California, Berkeley, USA

Université de Montréal, Canada

San Francisco State University, USA

Oxford University, UK

Hebrew University, Israel

University of Virginia, USA

Yale University, USA

University of Tokyo, Japan

Princeton University, USA

Northwestern University, USA

Harvard University, USA

École Polytechnique Fédérale de Lausanne
Susan Montgomery

Shigefumi Mori

Raman Parimala

Jonathan Pila

Victor Reiner

Karl Rubin

Peter Sarnak

Joseph H. Silverman

Michael Singer

Vasudevan Srinivas

J. Toby Stafford

Bernd Sturmfels

Richard Taylor

Ravi Vakil

Michel van den Bergh

Marie-France Vignéras

Kei-Ichi Watanabe

Efim Zelmanov

Shou-Wu Zhang
University of Southern California, USA

RIMS, Kyoto University, Japan

Emory University, USA

University of Oxford, UK

University of Minnesota, USA

University of California, Irvine, USA

Princeton University, USA

Brown University, USA

North Carolina State University, USA

Tata Inst. of Fund. Research, India

University of Michigan, USA

University of California, Berkeley, USA

Harvard University, USA

Stanford University, USA

Hasselt University, Belgium

Université Paris VII, France

Nihon University, Japan

University of California, San Diego, USA

Princeton University, USA

PRODUCTION

production@msp.org

Silvio Levy, Scientific Editor

See inside back cover or msp.org/ant for submission instructions.

The subscription price for 2014 is US $\$ 225 /$ year for the electronic version, and $\$ 400 /$ year $(+\$ 55$, if shipping outside the US) for print and electronic. Subscriptions, requests for back issues and changes of subscribers address should be sent to MSP.

Algebra \& Number Theory (ISSN 1944-7833 electronic, 1937-0652 printed) at Mathematical Sciences Publishers, 798 Evans Hall \#3840, c/o University of California, Berkeley, CA 94720-3840 is published continuously online. Periodical rate postage paid at Berkeley, CA 94704, and additional mailing offices.

ANT peer review and production are managed by EditFLOW ${ }^{\circledR}$ from Mathematical Sciences Publishers.

\section{PUBLISHED BY}

- mathematical sciences publishers

nonprofit scientific publishing

http://msp.org/

(C) 2014 Mathematical Sciences Publishers 


\section{Algebra \& Number Theory}

Volume $8 \quad$ No. $2 \quad 2014$

Large self-injective rings and the generating hypothesis

LEIGH SHEPPERSON and NEIL STRICKLAND

On lower ramification subgroups and canonical subgroups

303

SHIN HATTORI

Wild models of curves

DINO LORENZINI

Geometry of Wachspress surfaces

COREY IRVING and HAL SCHENCK

Daniel Goldstein, Robert M. GuRAlnick, Mark L. Lewis, AleXander Moretó, Gabriel Navarro and Pham HuU TieP

The homotopy category of injectives

AMNON NEEMAN

Essential dimension of spinor and Clifford groups

Vladimir Chernousov and AleXANDER MERKurJeV

On Deligne's category $\underline{\operatorname{Rep}}^{a b}\left(S_{d}\right)$

JONATHAN COMES and VICTOR OSTRIK

Algebraicity of the zeta function associated to a matrix over a free group algebra

CHRISTIAN KASSEL and Christophe REUTENAUER 